\title{
BOUNDARY LIMIT THEORY FOR FUNCTIONAL LOCAL TO UNITY REGRESSION
}

\author{
ANNA BYKHOVSKAYA AND PETER C. B. PHILLIPS
}

\begin{abstract}
This paper studies functional local unit root models (FLURs) in which the autoregressive coefficient may vary with time in the vicinity of unity. We extend conventional local to unity (LUR) models by allowing the localizing coefficient to be a function which characterizes departures from unity that may occur within the sample in both stationary and explosive directions. Such models enhance the flexibility of the LUR framework by including break point, trending, and multi-directional departures from unit autoregressive coefficients. We study the behavior of this model as the localizing function diverges, thereby determining the impact on the time series and on inference from the time series as the limits of the domain of definition of the autoregressive coefficient are approached. This boundary limit theory enables us to characterize the asymptotic form of power functions for associated unit root tests against functional alternatives. Both sequential and simultaneous limits (as the sample size and localizing coefficient diverge) are developed. We find that asymptotics for the process, the autoregressive estimate, and its $t$ statistic have boundary limit behavior that differs from standard limit theory in both explosive and stationary cases. Some novel features of the boundary limit theory are the presence of a segmented limit process for the time series in the stationary direction and a degenerate process in the explosive direction. These features have material implications for autoregressive estimation and inference which are examined in the paper.

Keywords and phrases: Boundary asymptotics, Functional local unit root; Local to unity; Sequential limits; Simultaneous limits; Unit root model

JEL Classification: C22, C65
\end{abstract}

\section{INTRODUCTION}

Time varying coefficient models have been extensively used in applied econometric work and provide a natural mechanism for a model to evolve over time. Various approaches have been studied in the literature, including early work originally published in 1971 by

\footnotetext{
Anna Bykhovskaya: Yale University

Peter C. B. Phillips: Yale University, University of Auckland, Southampton UniverSity, Singapore Management University

E-mail addresses: anna.bykhovskaya@yale.edu, peter.phillips@yale.edu.

Date: December 11, 2017.

Acknowledgements: We thank a referee for helpful comments and suggestions on the original version of the paper. Phillips acknowledges support from the NSF under Grant No. SES 12-58258 and from the Kelly Fund at the University of Auckland.
} 
Swamy (2012) on random coefficients, explicit parametric time series formulations (Harvey (1990)), time varying probability measures that are implied in Bayesian autoregressions (Phillips and Ploberger (1994)) and recent non-parametric work using kernel regression methods (Gao et al. (2008), Kristensen (2012), Giraitis et al. (2014)). The latter developments have emphasized the flexibility of nonparametric formulations and smooth transition approaches of capturing temporal coefficient evolution. Both these ideas have been used in practical econometric work. They also provide a mechanism for modeling nonstationarity through the vehicle of nearly integrated time series without insisting on a fixed local unit root structure, thereby accommodating departures from unity in both stationary and explosive directions that can evolve over time ( Phillips and Yu (2011); Greenaway-McGrevy and Phillips (2016)). Such models are called functional local unit root models (FLURs). They were recently studied in Bykhovskaya and Phillips (2017) in the context of point optimal unit root tests, showing how different the power envelope can be when the departures from a unit root are time varying.

The advantage of FLUR models compared to the standard local unit root (LUR) model (Phillips (1987), Chan and Wei (1987)) is that they explicitly allow the autoregression coefficient $\theta$ to vary with time, while retaining proximity to a unit root. This proximity is achieved through the specification $\theta_{t n}=1+\frac{c(t / n)}{n}$ which involves a localizing time varying coefficient function $c\left(\frac{t}{n}\right)$ dependent on the position of observation $t$ within the sample of size $n$. With this FLUR mechanism we can model economic and financial data that are well described in parts of the sample as unit root processes and yet subject to episodes of booms, busts, and recoveries at other times during the same sample period. Bykhovskaya and Phillips (2017) developed a limit theory for the FLUR process and analyzed some of the properties of functional point optimal unit root tests in comparison with standard (scalar) point optimal tests, showing that the latter delivers power that is often well below the optimal (functional) power envelope. This power deficiency of the standard point optimal test reflects the limitation of specifications that involve constant unidirectional departures from unity throughout the sample period when the data involves more complex forms of behavior, such as periods of intermittent departures from a unit root or periods of financial exuberance and collapse.

The present paper studies the same FLUR model as Bykhovskaya and Phillips (2017) and examines behavior as the localizing function $c\left(\frac{t}{n}\right)$ diverges, thereby determining the impact on the properties of the time series as the limits of the domain of definition of the autoregressive coefficient are approached. This boundary limit theory enables us to characterize the asymptotic form of power functions for associated unit root tests when the alternatives involve time varying functional forms of differing types. The results therefore extend the original work on boundary limit behavior of LUR models as a scalar localizing coefficient $c$ approaches the limits of its domain of definition. In contrast to this earlier 
work, the asymptotics for the process itself, the autoregressive estimate, and its associated $t$ statistic are all found to have boundary limit behavior that differs from standard limit theory in the explosive and stationary directions. Novel features of this boundary limit theory in the functional case are the presence of a segmented limit process for the time series in the stationary direction, a degenerate process in the explosive direction, and more complex function-dependent centering and standardization in the limit theory for the autoregressive coefficient estimator. These results differ markedly from the boundary limit theory that applies as the scalar localizing coefficient $c \rightarrow \pm \infty$ in the standard LUR model developed in Phillips (1987).

The paper is organized as follows. Some preliminary limit theory is given in the following section. Section 3 develops the boundary limit theory as the localized coefficient function $c(\cdot) \rightarrow \pm \infty$, which enables us to explore properties of the process at the limits of its domain of definition. Section 4 considers some mildly integrated (Phillips and Magdalinos (2007)) FLUR cases and the corresponding limit behavior at the boundary. This framework is of particular interest because it enables the analysis of asymptotics as $c(\cdot) \rightarrow \infty$ and $n \rightarrow \infty$ jointly, which has proved to be particularly useful in the study of uniform inference (Giraitis and Phillips (2006), Mikusheva (2007)). Implications of the findings are discussed in Section 5. Proofs are given in the Appendix.

\section{Preliminaries}

To fix ideas, we consider a time series generated by the model

$$
X_{t}=\theta_{t n} X_{t-1}+u_{t}, t=0,1, \ldots, n,
$$

where the autoregressive coefficient $\theta_{t n}=\exp \left(\frac{c(t / n)}{n}\right)=1+\frac{c(t / n)}{n}+o\left(\frac{1}{n}\right)$, the process $X_{t}$ is initialized at $X_{-1}=o_{p}(\sqrt{n})$ and the disturbances $u_{t}$ are zero mean stationary with

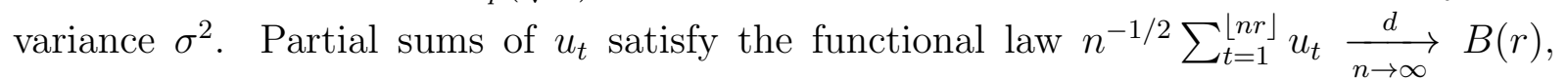
where $\underset{n \rightarrow \infty}{\stackrel{d}{\longrightarrow}}$ signifies weak convergence as $n \rightarrow \infty$, in the present case on the Skorohod space $D[0,1]$, and $B$ is Brownian motion with variance $\omega^{2}=\mathbb{E} u_{0}^{2}+2 \sum_{h=1}^{\infty} \mathbb{E} u_{0} u_{h}$, primitive conditions for which are widely available (e.g., Phillips and Solo (1992)).

Time series generated by (1) are near integrated arrays with a localizing coefficient function $c\left(\frac{t}{n}\right)$ that allows for variation in the autoregressive coefficient according to the position in the sample while retaining proximity to unity. The model is therefore a time varying coefficient model in the vicinity of unity. It is a particularly useful framework for studying the effects of departures from simple unit root and LUR models to more complex time series behavior.

Bykhovskaya and Phillips (2017) show that upon standardization the process $X_{t}$ satisfies the functional law given in the lemma below with a Gaussian limit process which 
extends the usual limit theory for LUR time series. This functional law embodies the impact in the limit process of the functional localizing coefficient that is present in (1). It is used extensively throughout the paper. In what follows, we confine attention to finite variance processes, Gaussian limit processes and functionals of them. Certain extensions to infinite variance processes and stable limit processes are also possible but will not be pursued here.

Lemma 1 (Bykhovskaya and Phillips (2017)). If $X_{t}$ is defined by (1), then

$$
n^{-\frac{1}{2}} X_{\lfloor n r\rfloor} \underset{n \rightarrow \infty}{\stackrel{d}{\longrightarrow}} F_{c}(r):=\omega K_{c}(r)=\omega \int_{0}^{r} e^{\int_{s}^{r} c(a) d a} d W(s),
$$

where $W$ is standard Brownian motion and $K_{c}(r)$ satisfies the following nonlinear diffusion equation $d K_{c}(r)=c(r) K_{c}(r) d r+d W(r)$.

When $c(r)=c$ is constant throughout the sample, the limit process $K_{c}(r)$ reduces to the linear diffusion $\int_{0}^{r} e^{c(r-s)} d W(s)$ studied in Phillips (1987) and Chan and Wei (1987). In this LUR case, locally stationary and locally explosive time series occur according to the sign of $c$. Moreover, as $c \rightarrow \pm \infty$, LUR asymptotics of the centred least squares estimate of $\theta$ and its t ratio transition to the asymptotics for stationary and explosive time series. This transition provides a linkage between the limit theory for unit root, local unit root, stationary, and explosive models.

The following sections explore the behavior of certain functionals of $K_{c}(r)$ as $c(\cdot)$ approaches the limits of its domain of definition. This limit behavior is of interest because it describes the links between near-integrated time series of the FLUR class (1) and time series that transition between unit root, stationary, and explosive processes. Correspondingly, this limit theory captures the limiting forms of the power functions of unit root tests at the limits of the domain of definition of $c(r)$. In particular, when $c(\cdot) \rightarrow \pm \infty$, the limit theory determines whether unit root tests are consistent against certain functional alternatives to a unit root in both stationary and explosive directions and the role of functional shape in determining power.

The fixed coefficient autoregression can be viewed as a special case of the FLUR model with $c(r)=n \times$ const and const $\neq 0$. Thus, taking limits as $c(\cdot) \rightarrow \pm \infty$ may be viewed as delivering an approximate route to standard autoregression at least when $c(r) \neq 0$ for all $r$. Our primary interest in the present paper, however, concerns cases in which $c(r)=0$ and $c(r) \neq 0$ occur over complementary subperiods, thereby allowing for finite sample episodes of unit root and FLUR behavior within the same sample of observations. Boundary limit theory as $c(\cdot) \rightarrow \pm \infty$ then reveals the asymptotic impact of these subperiod extremes of stationarity and explosiveness. As usual in multidimensional asymptotics (Phillips and Moon, 1999), there are two possibilities: sequential and simultaneous limit theory. The following section considers sequential limits, when first $n$ goes to infinity and then $c(\cdot)$ 
goes to either plus or minus infinity. This limit theory extends to the FLUR environment the sequential asymptotics for LUR models (Phillips (1987)). Later we develop pathwise joint limit theory that provides simultaneous asymptotics under the condition that $k_{n}=$ $n / c \rightarrow \pm \infty$. These pathwise joint limits implement in the FLUR setting the concept of mild integration/mild explosiveness that was developed in Phillips and Magdalinos (2007).

Our discussion concentrates on the use of ordinary least squares (OLS) regression on (1). This focus is useful because the limit theory (both as $n \rightarrow \infty$ and the boundary limit theory where $c(\cdot) \rightarrow \pm \infty$ ) shows the impact on the standard OLS estimator and associated tests of episodes of near integration that take a general functional form of departure from unity. The theory also provides asymptotic power function behavior of unit root tests against such general alternatives in which there may still exist periods of unit root behavior. The presence of near integration in the generating process of $X_{t}$ is unknown and, in practice, unknowable given that the localizing coefficient is not consistently estimable. It is therefore of wide interest to understand the properties of standard OLS regression under general functional departures from unity. Issues of confidence interval construction and the potential for uniform inference in the presence of such function departures are considered in other ongoing work (Phillips (2017)).

\section{Limit Distributions AS $c(\cdot) \rightarrow \pm \infty$}

One advantage of the FLUR specification is that use of a localizing function $c(\cdot)$ rather than a constant $c$ in characterizing departures from unity accommodates subsample unit root behavior whenever the localizing function is zero. As might therefore be expected, asymptotic behavior can vary considerably depending on the specific form and properties of $c(\cdot)$. Regions of zero and non-zero values of $c(\cdot)$ turn out to be particularly important in the limit theory as they switch unit root behavior on and off during the sample. The impact of such switches are naturally magnified as $c(\cdot)$ approaches the limits of its domain of definition.

This section investigates the impact of switching behavior on the limit theory by considering localizing functions $c(\cdot)$ that switch from zero over some interval $\left[0, r_{1}\right]$ to non-zero values over $\left(r_{1}, r_{2}\right]$ and switch back to zero on $\left(r_{2}, 1\right]$ for $0<r_{1}<r_{2}<1$. This specification enables us to study rather general forms of subperiod near integration and near explosiveness in the data on the asymptotic behavior of FLUR autoregressions.

To capture boundary behavior we model passage to the limit $c(\cdot) \rightarrow \pm \infty$ via the specification $c(t)=c \cdot f(t)$, where $c$ is a scalar that passes to $\pm \infty$, and $f(\cdot)$ is a given piecewise continuous, bounded (and, thus, integrable) function of constant sign, designating the direction of the departure from unity. The model therefore has the form (1) with time 
varying coefficient

$$
\theta_{t n}= \begin{cases}1, & t \leq \tau_{1}=\left\lfloor n r_{1}\right\rfloor \\ 1+\frac{c f(t / n)}{n}, & t \in\left(\tau_{1}, \tau_{2}\right], \tau_{2}=\left\lfloor n r_{2}\right\rfloor \\ 1, & t>\tau_{2},\end{cases}
$$

where the function $f(\cdot)$ is zero outside $\left[r_{1}, r_{2}\right]$, integrable on $\left[r_{1}, r_{2}\right]$, and strictly positive on $\left(r_{1}, r_{2}\right)$. Simple examples include level partial departures from unity such as $f(x)=$ $\mathbf{1}\left\{x \in\left[r_{1}, r_{2}\right]\right\}$, triangular departures such as $f(x)=\left(x-r_{1}\right) \mathbf{1}\left\{x \in\left[r_{1}, \bar{r}\right]\right\}+\frac{\bar{r}-r_{1}}{\bar{r}-r_{2}}(x-$ $\left.r_{2}\right) \mathbf{1}\left\{x \in\left(\bar{r}, r_{2}\right]\right\}$ or various smooth variants of such functions.

Using (3) and iterative back-substitution for $X_{t}$ in (1) we have the solution

$$
\begin{aligned}
X_{t} & =\left\{X_{-1}+\sum_{s=0}^{t} u_{s}\right\} \mathbf{1}\left\{t \leq \tau_{1}\right\}+\left\{\sum_{j=0}^{t-\tau_{1}-1} u_{t-j} \prod_{l=0}^{j-1} \theta_{t-l, n}+X_{\tau_{1}} \prod_{l=0}^{t-\tau_{1}-1} \theta_{t-l, n}\right\} \mathbf{1}\left\{t \in\left(\tau_{1}, \tau_{2}\right]\right\} \\
& +\left\{\sum_{s=\tau_{2}+1}^{t} u_{s}+X_{\tau_{2}}\right\} \mathbf{1}\left\{t>\tau_{2}\right\},
\end{aligned}
$$

showing the presence of two periods of unit root behavior that occur at the start and at the end of the sample. Intermediate between these periods is an episode in which the process evolves with a time varying parameter in the vicinity of unity.

The following subsections examine the limit behavior of the $X_{t}$ process and the standard OLS autoregressive coefficient estimate and its associated $t$-ratio centered on unity, which are defined as usual, viz.,

$$
\hat{\theta}_{O L S}=\frac{\sum_{t=0}^{n} X_{t-1} X_{t}}{\sum_{t=0}^{n} X_{t-1}^{2}}, \quad t_{\hat{\theta}_{O L S}}=\frac{\hat{\theta}_{O L S}-1}{\hat{s}_{\hat{\theta}_{O L S}}}=\frac{\sqrt{\sum_{t=0}^{n} X_{t-1}^{2}}\left(\hat{\theta}_{O L S}-1\right)}{\sqrt{\frac{1}{n} \sum_{t=0}^{n}\left(X_{t}-\hat{\theta}_{O L S} X_{t-1}\right)^{2}}} .
$$

As indicated above, the large $n$ and large $|c|$ boundary limit theory reveal the impact of episodes of functional form departures from unity on standard estimation and test procedures, which help in analyzing the asymptotic power of unit root tests against alternatives where subperiods of unit root behavior persist in the sample data.

We examine limit behavior in two boundary cases corresponding to stationary $(c<0)$ and explosive $(c>0)$ departures from unity as the extent of the departure $|c| \rightarrow \infty$ while retaining the same functional form for the localizing coefficient function $f(\cdot)$. Sequential limits are used in the following three theorems and these limits employ the notation $(-c, n)_{\text {seq }} \rightarrow \infty$ for limits in which $n \rightarrow \infty$ followed by $c \rightarrow-\infty$, and similarly $(c, n)_{\text {seq }} \rightarrow$ $\infty$ denotes limits in which $n \rightarrow \infty$ followed by $c \rightarrow \infty$. The notation $\frac{d}{(-c, n)_{s e q} \rightarrow \infty}$ signifies sequential weak convergence on $D[0,1]$ or $\mathbb{R}$ according to the context. We look first at the stationary boundary. 
BOUNDARY LIMIT THEORY FOR FUNCTIONAL

\subsection{Sequential limits when $c(\cdot) \rightarrow-\infty$.}

Theorem 1. The standardized process $\frac{X_{\lfloor n r\rfloor}}{\sqrt{n}}$, the least squares estimate $\hat{\theta}_{O L S}$ of $\theta_{t n}$, and the associated $t$-ratio centered on unity have the following limit behavior under sequential limits in which $n \rightarrow \infty$ followed by $c \rightarrow-\infty$ :

$$
\begin{gathered}
\frac{X_{\lfloor n r\rfloor}}{\sqrt{n}} \frac{d}{(-c, n)_{s e q} \rightarrow \infty} B^{a}(r) \times \mathbf{1}\left\{0 \leq r \leq r_{1}\right\}+B^{b}(r) \times \mathbf{1}\left\{r_{2}<r \leq 1\right\}, \\
n\left(\hat{\theta}_{O L S}-1-\frac{\int_{0}^{1} c f(r) F_{c}^{2}(r) d r}{n \int_{0}^{1} F_{c}^{2}(r) d r}\right) \\
\underset{(-c, n)_{s e q} \rightarrow \infty}{\longrightarrow} \frac{\int_{0}^{r_{1}} B^{a}(r) d B^{a}(r)+\int_{r_{2}}^{1} B^{b}(r) d B^{b}(r)+\lambda}{\int_{0}^{r_{1}} B^{a}(r)^{2} d r+\int_{r_{2}}^{1} B^{b}(r)^{2} d r}, \\
\underset{(-c, n)_{s e q} \rightarrow \infty}{\longrightarrow} \frac{\int_{r_{2}}^{1} B^{b}(r) d B^{b}(r)+\lambda-\frac{1}{2} \omega^{2} r_{2}}{\int_{0}^{r_{1}} B^{a}(r)^{2} d r+\int_{r_{2}}^{1} B^{b}(r)^{2} d r}, \\
\underset{t_{\hat{\theta}_{O L S}}}{\stackrel{d}{(-c, n)_{s e q} \rightarrow \infty}} \frac{\int_{r_{2}}^{1} B^{b}(r) d B^{b}(r)+\lambda-\frac{1}{2} \omega^{2} r_{2}}{\sigma\left(\int_{0}^{r_{1}} B^{a}(r)^{2} d r+\int_{r_{2}}^{1} B^{b}(r)^{2} d r\right)^{1 / 2}},
\end{gathered}
$$

where $F_{c}(r)=\omega \int_{0}^{r} e^{e_{s}^{r} c(a) d a} d W(s), B^{a}$ and $B^{b}$ are independent Brownian motions with variance $\omega^{2}$, and $\lambda=\frac{1}{2}\left(\omega^{2}-\sigma^{2}\right)$.

The limit (4) is a segmented Brownian motion process which vanishes on the interval where $f(r)>0$ and has independent Brownian motion segments on the intervals $\left[0, r_{1}\right]$ and $\left(r_{2}, 1\right]$ where the localizing function $f(r)=0$.

When the one-sided long run covariance $\lambda=0$, the limit distribution of the randomcentred least squares estimate $\hat{\theta}_{O L S}$ given by (5) has the simpler form

$$
n\left(\hat{\theta}_{O L S}-1-\frac{\int_{0}^{1} c f(r) F_{c}^{2}(r) d r}{n \int_{0}^{1} F_{c}^{2}(r) d r}\right) \underset{(-c, n)_{s e q} \rightarrow \infty}{\longrightarrow} \frac{\int_{0}^{r_{1}} B^{a}(r) d B^{a}(r)+\int_{r_{2}}^{1} B^{b}(r) d B^{b}(r)}{\int_{0}^{r_{1}} B^{a}(r)^{2} d r+\int_{r_{2}}^{1} B^{b}(r)^{2} d r},
$$

which we call a segmented unit root limit distribution because of the excision of the subperiod $\left[r_{1}, r_{2}\right]$ in the numerator and denominator integrals. When $r_{1}=1$ or $r_{2}=0$ there is no episode of near integration and this distribution corresponds to the standard unit root distribution. As is apparent from the form of (5), centering of the OLS estimate $\hat{\theta}_{O L S}$ involves the random element $\int_{0}^{1} c f(r) F_{c}^{2}(r) d r / \int_{0}^{1} F_{c}^{2}(r) d r$. This random centering takes account of the varying departures from unity that are involved in the FLUR model 
and is the natural analogue of the constant centering that is employed when the localizing effect is constant and $c(\cdot)=c$. In that case the stochastic component cancels in the adjustment term. As shown in the proof, when $c \rightarrow-\infty$, random centering can be replaced by unit centering but with a corresponding impact on the limit distribution, as evident in the form of the limit density for the (non-random) unit-centred density given in (6).

As can be seen from (5) and (6), the use of random centering leads to asymptotics that are less left-shifted because of the decomposition

$$
\begin{aligned}
& \frac{\int_{r_{2}}^{1} B^{b}(r) d B^{b}(r)+\lambda-\frac{1}{2} \omega^{2} r_{2}}{\int_{0}^{r_{1}} B^{a}(r)^{2} d r+\int_{r_{2}}^{1} B^{b}(r)^{2} d r} \\
& =\frac{\int_{r_{2}}^{1} B^{b}(r) d B^{b}(r)+\lambda-\frac{1}{2} \omega^{2} r_{2}}{\int_{0}^{r_{1}} B^{a}(r)^{2} d r+\int_{r_{2}}^{1} B^{b}(r)^{2} d r}-\frac{\frac{1}{2}\left(\omega^{2}\left(r_{2}-r_{1}\right)+B^{a}\left(r_{1}\right)^{2}\right)}{\int_{0}^{r_{1}} B^{a}(r)^{2} d r+\int_{r_{2}}^{1} B^{b}(r)^{2} d r}
\end{aligned}
$$

where a strictly positive random variable is subtracted. Alternatively, this featue of the limit theory can be deduced from the fact that for $c<0$, we have $\frac{\int_{0}^{1} c f(r) F_{c}^{2}(r) d r}{n \int_{0}^{1} F_{c}^{2}(r) d r}<0$, so that a positive random variable is added to the centred statistic $\hat{\theta}_{O L S}-1$.

Figure 1 shows the asymptotic density of the unit-centred (6) OLS estimate $n\left(\hat{\theta}_{O L S}-1\right)$ for $\lambda=0, \omega=1, r_{1}=1 / 3, r_{2}=2 / 3$ along with the densities of the corresponding segmented (5) and standard unit root densities for comparison. All these densities are skewed and have a typical unit root distributional shape with a long left tail. The standard unit root density has the largest skewness and most dispersion, the segmented unit root has the least skewness and dispersion, and the unit-centred OLS density is the most left shifted, showing how miscentering accentuates the downward bias in the limit distribution. These shapes become more distinct as $r_{1} \rightarrow 0$ and $r_{2} \rightarrow 1$.

These results reveal the substantial impact that FLUR specifications have on near unit root limit theory. In the standard LUR model as the localizing scalar parameter $c \rightarrow-\infty$, the correctly centred and scaled OLS estimate has a boundary limit normal distribution that correctly reproduces the standard stationary case limit theory. This uniformity in the limit theory plays an important role in the construction of uniform inference procedures (Mikusheva (2007), Mikusheva (2012), Phillips (2014)). In the FLUR model, the boundary limit theory has greater complexity that reflects features of the localizing coefficient function even in the limit as $c \rightarrow-\infty$ and this no longer generally reproduces the stationary limit theory. In particular, the form of the localizing coefficient function plays a role in correct centering of the distribution, when this centering is random. When there are episodes of unit root behavior in the process, these episodes continue to impact the limit theory at the boundary. 


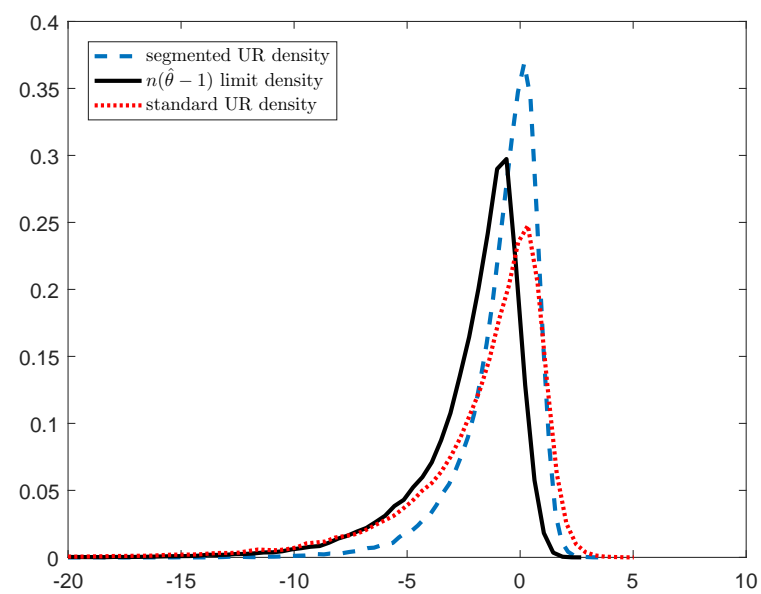

FiguRE 1. Densities of the OLS estimate, unit root, and segmented unit root for $\lambda=0, \omega=1, r_{1}=1 / 3, r_{2}=2 / 3$.

The boundary limits (6) and (7) show that both the coefficient-based and t-ratio unit root tests are inconsistent against the alternative of breaks that involve subperiods of stationarity. When there are subperiods of unit root behavior in the data, the tests do not diverge and therefore fail to detect the existence of stationary episodes in the sample with probability one as $c \rightarrow-\infty$.

\subsection{Sequential limits when $c(\cdot) \rightarrow \infty$.}

Theorem 2. Upon appropriate standardization, the process $\frac{X_{\lfloor n r\rfloor}}{\sqrt{n}}$, the least squares estimate $\hat{\theta}_{O L S}$ of $\theta_{t n}$, and the associated $t$-ratio centered on unity have the following limit behavior under sequential limits in which $n \rightarrow \infty$ followed by $c \rightarrow \infty$ :

$$
\begin{gathered}
\left(e^{-c \int_{r_{1}}^{r_{2}} f(a) d a}\right) \frac{X_{\lfloor n r\rfloor}}{\sqrt{n}} \frac{d}{(c, n)_{s e q} \rightarrow \infty} B^{a}\left(r_{1}\right) \times 1\left\{r_{2} \leq r \leq 1\right\}, \\
e^{c \int_{r_{1}}^{r_{2}} f(a) d a} n\left(\hat{\theta}_{O L S}-1-\frac{\int_{0}^{1} c f(r) F_{c}^{2}(r) d r}{n \int_{0}^{1} F_{c}^{2}(r) d r}\right) \frac{d}{(c, n)_{s e q} \rightarrow \infty} \frac{1}{\sqrt{r_{1}\left(1-r_{2}\right)}} \mathcal{C}, \\
n\left(\hat{\theta}_{O L S}-1\right) \underset{(c, n)_{s e q} \rightarrow \infty}{\longrightarrow} \frac{1}{2\left(1-r_{2}\right)}, \\
e^{-c \int_{r_{1}}^{r_{2}} f(a) d a} t_{\hat{\theta}_{O L S}} \frac{d}{(c, n)_{s e q} \rightarrow \infty} \frac{\omega \sqrt{r_{1}}}{2 \sigma \sqrt{1-r_{2}}}|\mathcal{N}(0,1)|,
\end{gathered}
$$

where $B^{a}$ is a Brownian motion with variance $\omega^{2}$, and $\mathcal{C}$ is standard Cauchy. 
As shown from (46) in the proof of the theorem, the limit behavior of $X_{\lfloor n r\rfloor}$ as $n \rightarrow \infty$ has the form

$$
\frac{X_{\lfloor n r\rfloor}}{\sqrt{n}} \rightarrow F_{c}(r)= \begin{cases}B^{a}(r), & r \leq r_{1}, \\ J_{c}\left(r ; r_{1}\right), & r_{1}<r \leq r_{2}, \\ B^{b}(r)+J_{c}\left(r_{2} ; r_{1}\right), & r_{2}<r \leq 1 ;\end{cases}
$$

with independent Brownian motion components $B^{a} \perp B^{b}$ and nonlinear diffusion component

$$
J_{c}\left(r ; r_{1}\right)=\int_{0}^{r} e^{c \int_{s}^{r} f(a) d a} d B(s)=e^{c \int_{r_{1}}^{r} f(a) d a} B^{a}\left(r_{1}\right)+\int_{r_{1}}^{r} e^{c \int_{s}^{r} f(a) d a} d B(s) .
$$

As $c \rightarrow \infty$ the FLUR period of explosive behavior dominates through the presence of $J_{c}\left(r ; r_{1}\right)$. In particular, we have $X_{\lfloor n r\rfloor} \sim \sqrt{n} e^{c \int_{r_{1}}^{r} f(a) d a} B^{a}\left(r_{1}\right) \times \mathbf{1}\left\{r_{1} \leq r \leq r_{2}\right\}$ and, as in (8), $X_{\lfloor n r\rfloor} \sim \sqrt{n} e^{c \int_{r_{1}}^{r_{2}} f(a) d a} B^{a}\left(r_{1}\right) \times \mathbf{1}\left\{r_{2} \leq r \leq 1\right\}$, showing that the process diverges exponentially from the level $\sqrt{n} B^{a}\left(r_{1}\right)$ when $r>r_{1}$ Thus, over the period $\left[0, r_{1}\right]$ the standardized process $n^{-1 / 2} X_{\lfloor n r\rfloor}$ evolves as a unit root process and reaches the limit value $B^{a}\left(r_{1}\right)$ at $r=r_{1}$, at which point a break occurs in the generating mechanism and the process evolves in an explosive FLUR way that inflates the initial condition reached at $r=r_{1}$. This behavior continues until $r=r_{2}$ when unit root behavior re-commences but from an explosive initial condition given by $J_{c}\left(r_{2} ; r_{1}\right)$.

Correspondingly from $(9)$, an appropriately centred OLS estimate $\hat{\theta}_{O L S}$ has an explosive rate of convergence with rate $e^{c \int_{r_{1}}^{r_{2}} f(a) d a} \times n$. As in Theorem 1 , in this case, the centering is the random quantity $1+\int_{0}^{1} c f(r) F_{c}^{2}(r) d r / n \int_{0}^{1} F_{c}^{2}(r) d r$, which depends on the scale coefficient $c$, the function $f(\cdot)$ and the stochastic process $F_{c}(r)$. When $c \rightarrow \infty$, as shown in the proof of the theorem, the random component of this centering element approaches a constant, satisfying

$$
\frac{\int_{0}^{1} c f(r) F_{c}^{2}(r) d r}{\int_{0}^{1} F_{c}^{2}(r) d r} \underset{c \rightarrow \infty}{\stackrel{d}{\longrightarrow}} \frac{1}{2\left(1-r_{2}\right)}
$$

which leads to 10 . Thus, the coefficient-based unit root test $n\left(\hat{\theta}_{O L S}-1\right)=O_{p}(1)$ as $(c, n)_{s e q} \rightarrow \infty$ and therefore the test fails to diverge in the presence of an internal subperiod $\left(r_{1}, r_{2}\right]$ of explosive behavior in the FLUR model even at the boundary as $c \rightarrow \infty$. However, because the limit $\frac{1}{2\left(1-r_{2}\right)}>0$, the test does have non-trivial power at the boundary limit $c \rightarrow \infty$ and test power continues to increase as $r_{2} \rightarrow 1$ and the period of explosive behavior expands in the sample. This result provides analytic confirmation of the simulation results in Evans (1991) that showed how full sample period unit root tests performed poorly in the detection of periodically collapsing bubbles within sample. On the other hand, as shown in Phillips, $\mathrm{Wu}$ and $\mathrm{Yu}$ (2011), recursive unit root tests are consistent against such subperiod explosive alternatives. Recursive mechanisms of detection therefore have 
considerable advantage in this context, particularly in the presence of multiple bubbles (Phillips et al. (2015a), Phillips et al. (2015b) ).

Note that the limit theory $(9)$ for the centred OLS estimate is a scaled Cauchy distribution. Importantly, the scale coefficient $\left\{r_{1}\left(1-r_{2}\right)\right\}^{-1 / 2}$ diverges when either $r_{1} \rightarrow 0$ or $r_{2} \rightarrow 1$. This is explained by the fact that the convergence rate changes as the limits of the region of explosive behavior are reached. Such cases involve different asymptotics and different rates of convergence that account for the shape behavior of the functional coefficient $f(\cdot)$ at the limits of the domain of definition. They are reported in detail in ongoing work (Phillips (2017)) and one such result is given in Theorem 5 in the following section.

Interestingly, the asymptotic distribution of $\hat{\theta}_{O L S}$ is degenerate when centered on unity, as evident in the limit of the coefficient-based unit root test (10). Moreover, it is insufficient to simply recenter again using the constant $\frac{1}{2\left(1-r_{2}\right)}$. In fact, in the spirit of the proof of Theorem 2, we can show that when $f\left(r_{2}\right)>0$,

$$
c n\left(\hat{\theta}_{O L S}-1-\frac{1}{2\left(1-r_{2}\right) n}\right) \underset{(c, n)_{s e q} \rightarrow \infty}{\longrightarrow} \frac{-1}{4\left(1-r_{2}\right)^{2} f\left(r_{2}\right)},
$$

leading to a further degenerate distribution. The limit theory requires more precise approximation than (12) of the random centering that is present in (9). Our next theorem gives the correct non-degenerate asymptotics with deterministic centering.

Theorem 3. If $f(r) \geq C>0$ for all $r \in\left[r_{1}, r_{2}\right]$, the OLS estimate $\hat{\theta}_{O L S}$ has the following limiting Cauchy distribution after appropriate deterministic centering and scaling

$$
e^{c \int_{r_{1}}^{r_{2}} f(a) d a} n\left(\hat{\theta}_{O L S}-1-\frac{1-e^{-2 c \int_{r_{1}}^{r_{2}} f(a) d a}}{2 n\left(1-r_{2}+\int_{r_{1}}^{r_{2}} e^{-2 c \int_{r}^{r_{2}} f(a) d a} d r\right)}\right) \frac{d}{(c, n)_{s e q} \rightarrow \infty} \frac{\mathcal{C}}{\sqrt{3 r_{1}\left(1-r_{2}\right)}} .
$$

Intriguingly, Theorems 2 and 3 both lead to very similar Cauchy limit distributions. The only difference besides the recentering is the numerical coefficient $\frac{1}{\sqrt{3}}$ in Theorem 3 . The explanation for this simple scalar difference in the limits lies in the replacement of a random centering in (9) with accurate non-random centering in (13). The intuition is as follows: in Theorem 2 the centered statistic (9) has additional variability because of the random centering, which leads to larger dispersion in the Cauchy limit theory than in Theorem 3 where the centering is constant and dispersion decreases.

As is apparent in the proof of Theorem 3, the reason for the specific numerical constant $\frac{1}{\sqrt{3}}$ is that adjustment of the centering produces a demeaned Brownian motion in the limit theory rather than a Brownian motion, with a corresponding reduction in the variance of the numerator. In particular, in Theorem 2 the limit distribution is governed by the ratio 
$\frac{B^{b}(1)-B^{b}\left(r_{2}\right)}{\left(1-r_{2}\right) B^{a}\left(r_{1}\right)}$, whereas in Theorem 3 the governing ratio is

$$
\frac{B^{b}(1)-B^{b}\left(r_{2}\right)-\frac{1}{1-r_{2}} \int_{r_{2}}^{1} B^{b}(r) d r}{\left(1-r_{2}\right) B^{a}\left(r_{1}\right)} .
$$

The numerator in 14 is the demeaned form of the Brownian motion differential $B^{b}(1)-$ $B^{b}\left(r_{2}\right)$ and, correspondingly, the numerator variance reduces by the scale factor $\frac{1}{3}$, thereby leading to the numerical coefficient $1 / \sqrt{3}$ which appears in 13 .

\section{Mild FluR Models and Simultaneous Asymptotics}

The models considered so far in the paper all follow (1) with a time varying coefficient in the local vicinity of unity that has the generalized LUR form $\theta_{t n}=1+\frac{c(t / n)}{n}$, where $c(\cdot)$ is piecewise continuous and bounded function. In order to widen the vicinity of unity under analysis, this section considers coefficients $\theta_{t n}$ that pass to unity at a slower rate $O\left(k_{n}^{-1}\right)$ than $O\left(n^{-1}\right)$ where $k_{n} \rightarrow \infty$ and $\frac{k_{n}}{n} \rightarrow 0$. The autoregressive coefficients have the form

$$
\theta_{t n}=1+\frac{c(t / n)}{k_{n}}
$$

for some fixed function $c(\cdot)$. The formulation (15) falls in the class of mildly integrated/mildly explosive processes considered by Phillips and Magdalinos (2007). With this specification, wider departures from unity may be considered and it is possible to develop simultaneous asymptotics where the parameters $(|c(\cdot)|, n)$ may jointly pass to infinity. In this passage to infinity what matters is the ratio $k_{n}=\frac{n}{|c(\cdot)|}$ and, as above, we assume that $\frac{1}{k_{n}}+\frac{k_{n}}{n} \rightarrow 0$. For example, we may have $k_{n}=n^{\alpha}$ with $\alpha \in(0,1)$ or $k_{n}=n / \log n$.

Since the parameter setting (15) leads to autoregressive coefficients that are 'closer' asymptotically to the stationary zone than those of the FLUR model when $c(\cdot)<0$, it is convenient to develop the limit theory under stationary martingale difference errors $\left\{u_{t}\right\}$, a setting that is better suited to that context (Phillips and Magdalinos (2007); Giraitis and Phillips (2006)). Further, the functions $c(\cdot)$ permitted in this section correspond to some of those used in Phillips (2017). Specifically, we assume the function $c(\cdot) \neq 0$ on $[0,1]$. More precisely, in the explosive case where $c(\cdot)>0$ we assume $c(\cdot)$ is non-zero in some fixed regions of the origin and unity, so that the FLUR process is active in those regions. As will become clear in the following analysis, behavior in those regions is particularly important in the explosive case because they play a significant role in the behavior of the time series and, in consequence, the limit theory also. Finally, it is convenient to set the initial condition in (1) at $t=0$ and assume that $X_{0}=o_{p}\left(\sqrt{k_{n}}\right)$, which rules out initial condition effects, although this condition may be relaxed as in Phillips and Magdalinos (2009) with some attendant differences in the asymptotics. 
Models such as (1) with time varying autoregressive coefficients of the form (15) are mildly integrated/explosive functional local unit root (MIFLUR, MEFLUR) models. As will become clear, MEFLUR specifications lead to asymptotics where there are dependencies on specific function values, such as the origination and end point values $c(0), c(1)$ as well as the function $c(\cdot)$ over its full domain $[0,1]$.

For the MEFLUR case we also consider a second specification for the coefficient function in place of (15), viz.,

$$
\theta_{t n}=1+\frac{c\left(t / k_{n}\right)}{k_{n}}
$$

where $\theta_{t n}$ converges to unity at the slower rate $O\left(k_{n}^{-1}\right)$ and the time varying coefficient function $c(\cdot)$ is now scaled consonantly in $1 / k_{n}$ units rather than $1 / n$ units. In this case, the limit theory depends on the coefficient function $c(\cdot)$ over its entire domain, which is now $[0, \infty)$, and $c(\cdot)$ is accordingly assumed to be integrable over this domain.

4.1. Mildly Explosive FLUR. We start from the first specification 15 of $\theta_{t n}$ with $c(\cdot)>0$. Solving the system yields

$$
X_{t}=\theta_{t n} X_{t-1}+u_{t}=\sum_{j=1}^{t} e^{\frac{1}{k_{n}} \sum_{l=1}^{t-j} c\left(\frac{t-l+1}{n}\right)} u_{j}+e^{\frac{1}{k_{n}} \sum_{j=1}^{t} c\left(\frac{j}{n}\right)} X_{0} .
$$

When the localizing function $c(\cdot)>0$ the exponent $\sum_{j=1}^{t=\lfloor n r\rfloor} c\left(\frac{j}{n}\right)=O(n)$ diverges and the process needs re-scaling to account for the exponential coefficients of the shocks $u_{j}$ and to achieve non-degenerate asymptotics for $X_{t=\lfloor n r\rfloor}$. A convenient standardization of $X_{t}$ is $e^{\frac{1}{k_{n}} \sum_{j=1}^{t} c\left(\frac{j}{n}\right)}$ and this leads to the process $\tilde{X}_{t}$ which is defined below without employing array notation as

$$
\tilde{X}_{t}:=\frac{X_{t}}{e^{\frac{1}{k_{n}} \sum_{j=1}^{t} c\left(\frac{j}{n}\right)}}=\sum_{j=1}^{t} \frac{u_{j}}{e^{\frac{1}{k_{n}}} \sum_{l=t-j+1}^{t} c\left(\frac{t-l+1}{n}\right)}+X_{0},
$$

It is also convenient to construct the further standardized process with mirror image weighting function $\tilde{Y}_{t}:=\sum_{j=1}^{t} u_{j} e^{-\frac{1}{k_{n}} \sum_{\ell=j}^{t} c\left(\frac{\ell}{n}\right)}$, which is useful in the asymptotics that follow. These standardizations may be interpreted as functionalized versions of those that apply in mildly explosive model asymptotics (Phillips and Magdalinos (2007)).

The time series $\tilde{X}_{t}$ upweights early innovations $\left\{u_{j}: j=1,2, \ldots\right\}$ because of the smaller number of components that enter the summation $\sum_{\ell=t-j+1}^{t} c\left(\frac{t-\ell+1}{n}\right)$ in the exponent when the index $j$ is small. In a similar way, the time series $\tilde{Y}_{t}$ downweights early innovations $\left\{u_{j}: j=1,2, \ldots\right\}$ because of the larger number of components that enter the summation $\sum_{\ell=j}^{t} c\left(\frac{\ell}{n}\right)$ in the exponent. The process $\tilde{X}_{t}$ is therefore weighted in favor of the origination point of the observations, and $\tilde{Y}_{t}$, as a mirror image, is weighted in favor of the terminal 
point $t$ of the observations in the sum. The limit theory when $t \geq k_{n} \rightarrow \infty$ as $n \rightarrow \infty$ for these two standardized processes is given in the following theorem which reveals the importance of the endpoint conditions.

Theorem 4. If there exists $\varepsilon>0$ such that for all $r \in[0, \varepsilon] \cup[1-\varepsilon, 1], c(r) \geq C>0$, then for all $r \in(0,1]$ as $\frac{1}{k_{n}}+\frac{k_{n}}{n} \rightarrow 0$

$$
\begin{aligned}
& \frac{1}{\sqrt{k_{n}}} \tilde{X}_{\lfloor n r\rfloor} \underset{n \rightarrow \infty}{\stackrel{d}{\longrightarrow}} X_{c(0)}={ }_{d} \mathcal{N}\left(0, \frac{\sigma^{2}}{2 c(0)}\right), \\
& \frac{1}{\sqrt{k_{n}}} \tilde{Y}_{\lfloor n r\rfloor} \underset{n \rightarrow \infty}{\stackrel{d}{\longrightarrow}} Y_{c(1)}={ }_{d} N\left(0, \frac{\sigma^{2}}{2 c(1)}\right),
\end{aligned}
$$

and the limit variates $X_{c(0)}$ and $Y_{c(1)}$ are independent.

Thus, the standardized process $\tilde{X}_{\lfloor n r\rfloor} / \sqrt{k_{n}}$ tends for all $r \in(0,1]$ to the same random variable $X_{c(0)}$ whose distribution depends on the initial value $c(0)$ of the function $c(\cdot)$ and no other value. This provides a striking difference with the FLUR asymptotics shown in Lemma 1 where the whole function $c(\cdot)$ plays a role in the limiting stochastic process. The explanation is that since $c(\cdot)>0$ the time series $X_{t}$ is explosive, which means that initial shocks and initial conditions are magnified, as is apparent in the solution (17). More particularly, the coefficient $\exp \left\{\frac{1}{k_{n}} \sum_{l=1}^{t-j} c\left(\frac{t-l+1}{n}\right)\right\}$ is positive and increasing as $j$ decreases, so that in (17), the early shocks $\left\{u_{1}, u_{2}, \ldots\right\}$ have the largest coefficients and the greatest impact on $X_{t}$ comes from the early part of the series. Correspondingly, the standardized process $\tilde{X}_{t}$ in $(18)$ is dominated by the early $k_{n}$ elements of the series, which leads to the common central limit theorem given in Theorem 4 for $\frac{1}{\sqrt{k_{n}}} \tilde{X}_{\lfloor n r\rfloor}$, whose variance involves only $c(0)$ rather than the full function $c(\cdot)$. Analogous mirror-image intuition applies to the standardized process $\tilde{Y}_{\lfloor n r\rfloor} / \sqrt{k_{n}}$ where the terminal $k_{n}$ elements of the series lead to a common limit theory that depends on the end point $c(1)$ rather than the full function $c(\cdot)$.

Next we turn to the limit behavior of the autoregressive coefficient estimate $\hat{\theta}_{O L S}$. After suitable centering and scaling we obtain the asymptotic results reported in the following theorem. The first result uses random data-dependent centering. The second employs constant centering that depends on the function $c(\cdot)$ and in principle might be used to invert the distribution.

Theorem 5. Suppose $c(r)$ is integrable over $[0,1]$ and $c(r) \geq C>0$ for all $r \in[0, \varepsilon] \cup$ $[1-\varepsilon, 1]$ for some $\varepsilon>0$. Then, as $\frac{1}{k_{n}}+\frac{k_{n}}{n} \rightarrow 0$, the least squares estimate $\hat{\theta}_{O L S}$ has the following common limit theory with random and deterministic centering 


$$
\begin{gathered}
k_{n} e^{\frac{n}{k_{n}} \int_{0}^{1} c(a) d a}\left(\hat{\theta}_{O L S}-1-\frac{\sum_{t=1}^{n} \frac{c(t / n)}{k_{n}} X_{t-1}^{2}}{\sum_{t=1}^{n} X_{t-1}^{2}}\right) \underset{n \rightarrow \infty}{\longrightarrow} 2 \sqrt{c(0) c(1)} \mathcal{C}, \\
k_{n} e^{\frac{n}{k_{n}} \int_{0}^{1} c(a) d a}\left(\begin{array}{c}
\sum_{\text {OL }}^{n} c(t / n) e^{-\frac{2}{k_{n}} \sum_{j=t}^{n} c(j / n)} \\
\hat{\theta}_{n} \sum_{t=1}^{n} e^{-\frac{2}{k_{n}} \sum_{j=t}^{n} c(j / n)}
\end{array}\right) \underset{n \rightarrow \infty}{\stackrel{d}{\longrightarrow} 2 \sqrt{c(0) c(1)} \mathcal{C},}
\end{gathered}
$$

where $\mathcal{C}$ is a standard Cauchy variate.

The proof of Theorem 5 is lengthy and involves complex calculations which are given in full in the proof in the Appendix. Importantly, although (21) and (22) differ in terms of their respective centering, the limit distribution behavior remains the same. As shown in the proof, the random centering quantity $\frac{\sum_{t=1}^{n} c(t / n) X_{t-1}^{2}}{k_{n} \sum_{t=1}^{n} X_{t-1}^{2}}$ that appears in 21) equals $\frac{\sum_{t=1}^{n} c(t / n) e^{-\frac{2}{k_{n}} \sum_{j=t}^{n} c(j / n)}}{k_{n} \sum_{t=1}^{n} e^{-\frac{2}{k_{n}} \sum_{j=t}^{n} c(j / n)}}$ plus a random component of smaller order that is too small to affect centering but which, as shown in the proof, still comes into play in calculating the limit behavior of the rescaled and recentred estimator. As the result shows, the random centering can be replaced by a deterministic centering that is still dependent on the sample size but without affecting the Cauchy limit distribution or the scale of this distribution.

Further, as $\frac{1}{k_{n}}+\frac{k_{n}}{n} \rightarrow 0$ we find that

$$
\frac{\sum_{t=1}^{n} c(t / n) e^{-\frac{2}{k_{n}} \sum_{j=t}^{n} c(j / n)}}{\sum_{t=1}^{n} e^{-\frac{2}{k_{n}} \sum_{j=t}^{n} c(j / n)}}=\frac{\frac{1}{n} \sum_{t=1}^{n} c(t / n) e^{-\frac{2 n}{k_{n}} \frac{1}{n} \sum_{j=t}^{n} c(j / n)}}{\frac{1}{n} \sum_{t=1}^{n} e^{-\frac{2 n}{k_{n}} \frac{1}{n} \sum_{j=t}^{n} c(j / n)}} \rightarrow c(1)
$$

giving a simple limiting form of the re-centering element 23. This simple form cannot, however, be used directly in the limit theory (22) without consequence because the exponential rate of convergence $k_{n} e^{\frac{n}{k_{n}} \int_{0}^{1} c(a) d a}$ plays a key role in defining those components that drive the asymptotics, as explained in the proof of Theorem 5 . If the constant $c(1)$ were employed in centering the estimate $\hat{\theta}_{O L S}$, then the re-centred quantity $\left(\hat{\theta}_{O L S}-1-c(1) / k_{n}\right)$ diverges under exponential standardization; and appropriate standardization leads to a degenerate constant limit, as in Theorem 2 . 
Observing that $\theta_{n n}^{2}-1=\frac{2 c(1)}{k_{n}}\{1+o(1)\}, \theta_{1 n}^{2}-1=\frac{2 c(0)}{k_{n}}\{1+o(1)\}$ and $\Pi_{t=1}^{n} \theta_{t n} \sim$ $e^{k_{n}^{-1} \Sigma_{t=1}^{n} c\left(\frac{t}{n}\right)} \sim e^{\frac{n}{k_{n}} \int_{0}^{1} c(a) d a}$, we may write 22 in the equivalent form

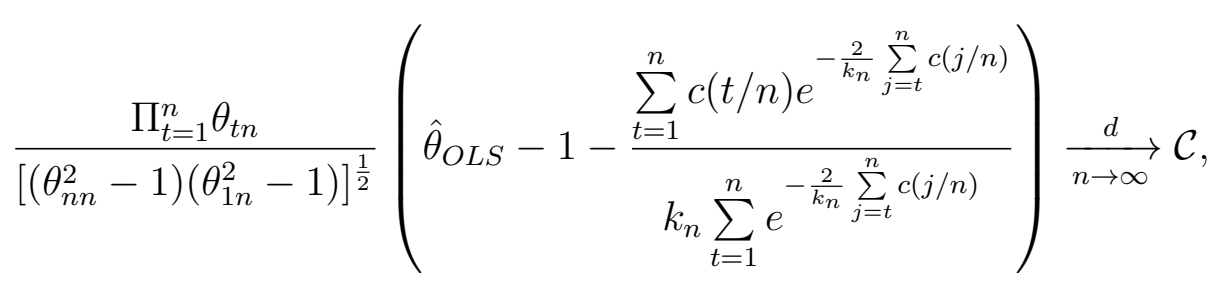

which is suggestive of earlier work in simpler cases of explosive process autoregression. In particular, 24 shows that, upon suitable standardization which in this cases relies on the time varying autoregressive coefficient, central limit theory holds for autoregressive estimation in the functional LUR with the same Cauchy limit theory as holds in (i) the fixed coefficient explosive case under Gaussian innovations with no invariance principle, and (ii) in the mildly explosive case under central limit theory (Phillips and Magdali$\operatorname{nos}(2007))$. Importantly, both the convergence rate and the centering depend on the functional coefficient $c(\cdot)$ throughout the $[0,1]$ interval.

In the special case where $c(\cdot)=c>0$ is constant and $\theta_{t n}=1+\frac{c}{k_{n}}=: \theta_{n}$ for all $t=1, \ldots, n, 24$ reduces to the form

$$
\frac{\theta_{n}^{n}}{\theta_{n}^{2}-1}\left(\hat{\theta}_{O L S}-1-\frac{c}{k_{n}}\right) \underset{n \rightarrow \infty}{\stackrel{d}{\longrightarrow}} \mathcal{C},
$$

the result obtained originally in Phillips and Magdalinos (2007) for a constant mildly explosive process $X_{t}$ with autoregressive coefficient $\theta=1+\frac{c}{k_{n}}$ for which $\theta_{n}^{2 n} \sim e^{\frac{2 n}{k_{n}} c}$. In that case the convergence rate of 25 is $\frac{\theta_{n}^{n}}{\theta_{n}^{2}-1} \sim \frac{k_{n}}{2 c} e^{\frac{n}{k_{n}} c}$. The limit theory given in 24 then specializes to this earlier theory as $\frac{1}{k_{n}}+\frac{k_{n}}{n} \rightarrow 0$.

In the present case, the primary implication of a functional localizing coefficient is to adjust the rate of convergence in 22 to embody the aggregate impact of the function $c(\cdot)$ over its full domain via the integral $\int_{0}^{1} c(a) d a$, so that the standardization factor is $k_{n} e^{\frac{2 n}{k_{n}} \int_{0}^{1} c(a) d a}$ rather than $k_{n} e^{\frac{2 n}{k_{n}} c}$. It is then the accumulative (mild FLUR) departure from unity that determines the convergence rate of the estimator $\hat{\theta}$. Interestingly, a secondary implication of the new limit theory in 22 is that the limit random variable $2 \sqrt{c(0) c(1)} \mathcal{C}$ depends explicitly on the behavior of the localizing function at the origination and termination dates via the pair $(c(0), c(1))$. This dependence is a consequence of the magnification of early and late shocks that takes place in the limiting process described above for a mildly explosive time series. The re-standardization by $\left[\left(\theta_{n n}^{2}-1\right)\left(\theta_{1 n}^{2}-1\right)\right]^{\frac{1}{2}}$ in $(24)$ adjusts for these initial and terminal effects and the dependence is eliminated.

Next consider the second specification $(16)$ with $c(\cdot)>0$. In this case, time is measured in $1 / k_{n}$ units rather than $1 / n$ units in the localizing coefficient function $c(\cdot)$ and since $\frac{n}{k_{n}} \rightarrow \infty$ the domain of the function is $[0, \infty)$, leading to the following limit theory for the 
standardized process when $c(\cdot)>0$ is integrable over $[0, \infty)]$

$$
\frac{1}{\sqrt{k_{n}}} \tilde{X}_{\lfloor n r\rfloor} \underset{n \rightarrow \infty}{\stackrel{d}{\longrightarrow}} X_{c, \infty}={ }_{d} \mathcal{N}\left(0, \sigma^{2} \int_{0}^{\infty} e^{-2 \int_{0}^{a} c(p) d p} d a\right) .
$$

The limit in (26) remains the same random variable for all values of $r$, just as in Theorem 4 above. But in the present case, as is clear from (26), the limit variance depends on function values $c(\cdot)>0$ over the full domain $[0, \infty)$ rather than the single function value $c(0)$ at the origin.

We may also consider the case where the standardized time series is measured in segments of length $O\left(k_{n}\right)$ rather than length $O(n)$. The framework then matches the usual FLUR model of Bykhovskaya and Phillips (2017) but over a much wider (infinite) domain. More specifically, when we focus on the process $X_{t}$ with $t=\left\lfloor k_{n} r\right\rfloor$ instead of $t=\lfloor n r\rfloor$, we have the following limit theory for the standardized process $\frac{1}{\sqrt{k_{n}}} \tilde{X}_{\left\lfloor k_{n} r\right\rfloor}$.

$$
\frac{1}{\sqrt{k_{n}}} \tilde{X}_{\left\lfloor k_{n} r\right\rfloor} \underset{n \rightarrow \infty}{\stackrel{d}{\longrightarrow}} X_{c, r}={ }_{d} \sigma \int_{0}^{r} e^{-\int_{0}^{a} c(p) d p} d W(a) .
$$

Importantly, in (27) the domain of $r$ is the half line $[0, \infty)$, thereby accommodating limit behavior of the process $X_{t}$ for $t \geq k_{n}$. Thus, the limit $X_{c, \infty}$ in (26) may be interpreted as the limit of the stochastic process $X_{c, r}$ as $r \rightarrow \infty$. The covariance kernel of $X_{c, r}$ is $\gamma(r, s)=\sigma^{2} \int_{0}^{\min (r, s)} e^{-2 \int_{0}^{a} c(p) d p} d a$, which reduces to the usual expression for the covariance kernel of a linear diffusion when $c(\cdot)$ is constant or to that of a Brownian motion when $c(\cdot)=0$.

4.2. Mildly stationary FLUR. We focus on the first specification (15). As shown below, this formulation leads to a limit process for a standardized version of $\left.X_{t=\lfloor n r}\right\rfloor$ where there is explicit dependence on the localizing function value $c(r)$ at the sample fraction $r$ corresponding to the sample point $t$. This outcome differs from $(19)$ and $(20)$ in the mildly explosive case where there is dependence on the end point values $c(0)$ and $c(1)$.

Suppose that $c(\cdot) \leq-C<0$. By $(17)$ we have the representation

$$
X_{t}=\sum_{j=1}^{t} e^{\frac{1}{k_{n}} \sum_{l=1}^{t-j} c\left(\frac{t-l+1}{n}\right)} u_{j}+e^{\frac{1}{k_{n}} \sum_{j=1}^{t} c(j / n)} X_{0} .
$$

and the following limit theory then holds for $X_{t}$ after suitable standardization.

Theorem 6. Given any fixed $r \in(0,1]$ for which $c(r) \leq-C<0$, then as $n \rightarrow \infty$

$$
\frac{1}{\sqrt{k_{n}}} X_{\lfloor n r\rfloor} \underset{n \rightarrow \infty}{\stackrel{f d d}{\longrightarrow}} X_{c}(r)={ }_{d} \mathcal{N}\left(0, \frac{\sigma^{2}}{-2 c(r)}\right), r \in(0,1] .
$$

Different values of $r$ lead to independent random variables. 
Importantly, (28) gives a finite dimensional limit distribution for each fixed $r$, not a functional law. This is signified in 28 by the affix 'fdd' in place of weak convergence over $[0,1]$. As the theorem indicates, the limit variates $X_{r}$ and $X_{s}$ are independent for all $r \neq s$. While the limit random variable $X_{r}$ exists for each fixed $r$, the limiting stochastic process $X_{r}$ on $r \in(0,1]$ has pathological path properties because the independence of arbitrarily adjacent components $X_{r}$ and $X_{s}$ implies a degree of local variability that is unrealizable.

\section{Some Implications of Boundary Limit Theory}

Local unit root limit theory enabled analysis of the power properties of unit root tests and helped explore the passage to stationary and explosive behavior by examining boundary behavior in the asymptotics. The LUR methodology has since been used extensively in the econometric analysis of tests in unit root models, cointegrated systems, and predictive regression. In FLUR models, departures from unity allow for functional, time dependent forms that vary over the sample period. Correspondingly, in FLUR specifications both the limit theory and the asymptotic power properties involve richer possibilities that accommodate realistic empirical situations where unit root behavior may be interrupted by episodes of near-stationary or near-explosive behavior in the data. The passages to stationary and explosive behavior at the boundary of functional specifications become similarly more complex and have implications for practical work with inference for time series data.

This paper has explored these implications in autoregressions when no allowance is made for time variation in the parameters in estimation and inference, as happens in practice when an investigator proceeds with parametric autoregression and standard testing procedures in ignorance of the greater complexity of the generating mechanism. In moving to the stationary boundary as might be expected in a time varying environment, the dominating component of the limit theory is any remaining nonstationary episode in the data. For the process itself, for the autoregressive estimate, and for unit root test statistics, the boundary asymptotics depend on the interval that defines this episode, leading to a form of segmented unit root limit theory. These results differ significantly from those of LUR boundary asymptotics which are well known to lead to standard normal asymptotics at the stationary boundary (Phillips (1987); Giraitis and Phillips (2006); Mikusheva (2007)). The implication is that functional departures from unity can have a major effect on limit theory and test performance.

Likewise, moving to the explosive boundary produces material changes in the asymptotics. In this case, the dominating component of the limit theory comes from the explosive episode in the data. Again, the boundary limit theory depends on the region that defines the episode. In the explosive direction, the boundary limit theory is centred in the 
explosive region. But while unit root tests have non-trivial power at the boundary they are not consistent, which partly explains the poor performance of right-sided unit root tests in the detection of periodic episodes of bubbles and the need for recursive regression methods of detection which have greater sensitivity to local departures from unity.

Functional local alternatives such as those considered here in the unit root context obviously have wider applications in statistical limit theory and power function analysis beyond those of unit root models, although there seems to have been little use or mention of them in the literature to date. They are also useful in the construction of functional point-optimal test procedures, where there are potential gains from the consideration of explicit functional alternatives rather than fixed alternatives. For instance, Bykhovskaya and Phillips (2017) examine some of the implications of functional departures for unit root testing with a focus on the properties of point optimal procedures. A further application of these boundary asymptotics that is relevant to empirical work is the impact of local time variation of the type considered here on uniform inference in autoregression. That subject is investigated in other ongoing work (Phillips (2017)). 


\section{Appendix}

The following Lemma relates to Lemma A of Phillips (2017) and provides a different proof of a version of parts (i) \& (ii) of that result. It is given here for convenient reference and is used throughout the remaining proofs in this Appendix.

Lemma 2. Suppose that functions $f(\cdot)$ and $g(\cdot)$ are piecewise continuous and bounded, and $f(s), g(s) \geq C>0$ for all $s \in\left[r_{1}, r_{2}\right]$. Then the following holds for any $r, r^{\prime} \in$ $\left[r_{1}, r_{2}\right], r^{\prime}>r, K>0$, as $c \rightarrow \infty:$

$$
\begin{gathered}
\int_{r}^{r^{\prime}} e^{-K c \int_{r}^{s} f(a) d a} g(s) d s=\frac{g\left(r_{+}\right)(1+o(1))}{K c f\left(r_{+}\right)}, \quad \int_{r}^{r^{\prime}} e^{-K c \int_{s}^{r^{\prime}} f(a) d a} g(s) d s=\frac{g\left(r_{-}^{\prime}\right)(1+o(1))}{K c f\left(r_{-}^{\prime}\right)}, \\
\mathbb{V} \int_{r}^{r^{\prime}} e^{-K c \int_{r}^{s} f(a) d a} g(s) d W(s)=\frac{g^{2}\left(r_{+}\right)(1+o(1))}{2 K c f\left(r_{+}\right)}, \mathbb{V} \int_{r}^{r^{\prime}} e^{-K c \int_{s}^{r^{\prime}} f(a) d a} g(s) d W(s)=\frac{g^{2}\left(r_{-}^{\prime}\right)(1+o(1))}{2 K c f\left(r_{-}^{\prime}\right)},
\end{gathered}
$$

where $W(s)$ is a standard Brownian motion, $f\left(r_{+}\right)\left(f\left(r_{-}\right)\right)$stands for the right (left) limit $\lim _{x \rightarrow r_{+}} f(x)\left(\lim _{x \rightarrow r_{-}} f(x)\right)$, and $\mathbb{V}$ stands for variance.

Proof. Fix $\delta<C$. There exists $\varepsilon>0$ such that $r+\varepsilon<r^{\prime}$ and $\left|f(s)-f\left(r_{+}\right)\right|<$ $\delta,\left|g(s)-g\left(r_{+}\right)\right|<\delta$ for all $s \in[r, r+\varepsilon]$. Then

$$
\begin{aligned}
& \int_{r}^{r+\varepsilon} e^{-K c \int_{r}^{s} f(a) d a} g(s) d s \\
& \in\left[\int_{r}^{r+\varepsilon} e^{-K c \int_{r}^{s}\left(f\left(r_{+}\right)+\delta\right) d a}\left(g\left(r_{+}\right)-\delta\right) d s, \int_{r}^{r+\varepsilon} e^{-K c \int_{r}^{s}\left(f\left(r_{+}\right)-\delta\right) d a}\left(g\left(r_{+}\right)+\delta\right) d s\right] \\
& =\left[\frac{g\left(r_{+}\right)-\delta}{K c\left(f\left(r_{+}\right)+\delta\right)}\left(1-e^{-K c\left(f\left(r_{+}\right)+\delta\right) \varepsilon}\right), \frac{g\left(r_{+}\right)+\delta}{K c\left(f\left(r_{+}\right)-\delta\right)}\left(1-e^{-K c\left(f\left(r_{+}\right)-\delta\right) \varepsilon}\right)\right],
\end{aligned}
$$

and

$$
0 \leq \int_{r+\varepsilon}^{r^{\prime}} e^{-K c \int_{r}^{s} f(a) d a} g(s) d s \leq \int_{r+\varepsilon}^{r^{\prime}} e^{-K c \int_{r}^{r+\varepsilon} f(a) d a} g(s) d s=e^{-K c \int_{r}^{r+\varepsilon} f(a) d a} \int_{r+\varepsilon}^{r^{\prime}} g(s) d s .
$$

Combining Eq. (29) and (30) and taking first the limit $c \rightarrow \infty$ and then $\delta \rightarrow 0$, we get

$$
c \int_{r}^{r^{\prime}} e^{-K c \int_{r}^{s} f(a) d a} g(s) d s=\frac{g\left(r_{+}\right)}{K f\left(r_{+}\right)}+o(1)=\frac{g\left(r_{+}\right)(1+o(1))}{K f\left(r_{+}\right)} .
$$

Thus, $\int_{r}^{r^{\prime}} e^{-K c \int_{r}^{s} f(a) d a} g(s) d s=\frac{g\left(r_{+}\right)(1+o(1))}{K c f\left(r_{+}\right)}$as $c \rightarrow \infty$.

The second asymptotic representation $\int_{r}^{r^{\prime}} e^{-K c \int_{s}^{r^{\prime}} f(a) d a} g(s) d s=\frac{g\left(r_{-}^{\prime}\right)(1+o(1))}{K c f\left(r_{-}^{\prime}\right)}$, as $c \rightarrow \infty$, follows in a similar way. Finally, using the formula for the variance of the Wiener integral 
and repeating previous arguments, we have

$$
\begin{gathered}
\mathbb{V} \int_{r}^{r^{\prime}} e^{-K c \int_{r}^{s} f(a) d a} g(s) d W(s)=\int_{r}^{r^{\prime}} e^{-2 K c \int_{r}^{s} f(a) d a} g^{2}(s) d s=\frac{g^{2}\left(r_{+}\right)(1+o(1))}{2 K c f\left(r_{+}\right)}, \\
\mathbb{V} \int_{r}^{r^{\prime}} e^{-K c \int_{s}^{r^{\prime}} f(a) d a} g(s) d W(s)=\int_{r}^{r^{\prime}} e^{-2 K c \int_{s}^{r^{\prime}} f(a) d a} g^{2}(s) d s=\frac{g^{2}\left(r_{-}^{\prime}\right)(1+o(1))}{2 K c f\left(r_{-}^{\prime}\right)} .
\end{gathered}
$$

\section{Proof of Theorem 1 .}

Proof. From (2) and with long run variance $\omega^{2}=\sum_{h=-\infty}^{\infty} \mathbb{E} u_{o} u_{h}$, we deduce that the limit process for $X_{t}$ as $n \rightarrow \infty$ has the following segmented form

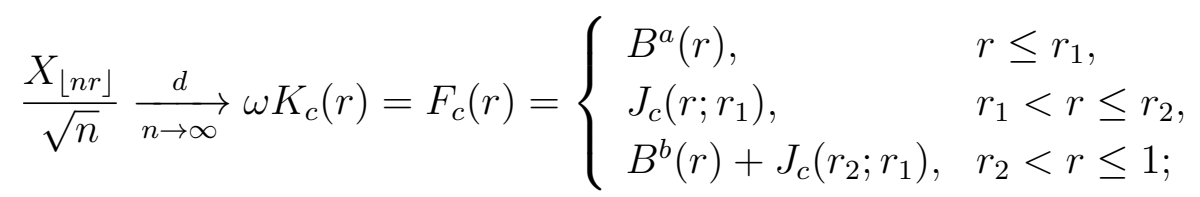

with $B^{a}, B^{b}=B M(\omega), B^{a} \perp B^{b}$ and

$$
\begin{aligned}
J_{c}\left(r ; r_{1}\right) & =\int_{0}^{r} e^{\int_{s}^{r} c f(a) d a} d B(s)=\int_{0}^{r_{1}} e^{\int_{r_{1}}^{r} c f(a) d a} d B(s)+\int_{r_{1}}^{r} e^{\int_{s}^{r} c f(a) d a} d B(s) \\
& =e^{c \int_{r_{1}}^{r} f(a) d a} B^{a}\left(r_{1}\right)+\int_{r_{1}}^{r} e^{c \int_{s}^{r} f(a) d a} d B(s) .
\end{aligned}
$$

Because $f(\cdot)$ is strictly positive on $\left(r_{1}, r_{2}\right)$, we know that $\int_{s}^{r} f(a) d a>0$ for all $s>$ $r_{1}, r>s$. Thus, $e^{c \int_{s}^{r} f(a) d a} \rightarrow 0$ monotonically in $s$ as $c \rightarrow-\infty$, so that $J_{c}\left(r ; r_{1}\right) \rightarrow 0$ as $c \rightarrow-\infty$ for $r \in\left(r_{1}, r_{2}\right]$. Therefore, the limit process $F_{c}(r)$ in (2) converges to $F_{-}(r)=B^{a}(r) \times \mathbf{1}\left\{0 \leq r \leq r_{1}\right\}+B^{b}(r) \times \mathbf{1}\left\{r_{2}<r \leq 1\right\}$.

We may now calculate the limit distribution of the OLS estimate $\hat{\theta}_{O L S}=\frac{\sum_{t=0}^{n} X_{t} X_{t-1}}{\sum_{t=0}^{n} X_{t-1}^{2}}$ in sequential asymptotics as $n$ passes to infinity followed by $c$ passing to minus infinity, which we write as $(-c, n)_{\text {seq. }} \rightarrow \infty$. Write

$$
n\left(\hat{\theta}_{O L S}-1\right)=\frac{\frac{1}{n} \sum_{t=0}^{n} X_{t-1} \Delta X_{t}}{\frac{1}{n^{2}} \sum_{t=0}^{n} X_{t-1}^{2}}=\frac{\frac{1}{n} \sum_{t=0}^{n} X_{t-1} u_{t}}{\frac{1}{n^{2}} \sum_{t=0}^{n} X_{t-1}^{2}}+\frac{\frac{1}{n} \sum_{t=0}^{n} \frac{c f(t / n)}{n} X_{t-1}^{2}}{\frac{1}{n^{2}} \sum_{t=0}^{n} X_{t-1}^{2}},
$$

and note from Lemma 1 that we may deduce the joint weak convergence

$$
\begin{gathered}
\frac{1}{n^{2}} \sum_{t=0}^{n} X_{t-1}^{2} \underset{n \rightarrow \infty}{\stackrel{d}{\longrightarrow}} \int_{0}^{1} F_{c}^{2}(r) d r \\
\frac{1}{n^{2}} \sum_{t=0}^{n} c f(t / n) X_{t-1}^{2} \underset{n \rightarrow \infty}{\stackrel{d}{\longrightarrow}} \int_{0}^{1} c f(r) F_{c}^{2}(r) d r=\omega^{2} \int_{r_{1}}^{r_{2}} c f(r) K_{c}^{2}(r) d r .
\end{gathered}
$$


To calculate the limit of $\frac{1}{n} \sum_{t=0}^{n} X_{t-1} u_{t}$ in the numerator of the first member of (31), first square Eq. (1), then sum over $t$, and scale by $n$, giving

$$
n^{-1} X_{n}^{2}=n^{-1} \sum_{t} u_{t}+2 n^{-1} \sum_{t} X_{t-1} u_{t}+2 n^{-1} \sum_{t} \frac{c f(t / n)}{n} X_{t-1}^{2}+O_{p}\left(n^{-0.5}\right),
$$

so that

$$
n^{-1} \sum_{t=0}^{n} X_{t-1} u_{t} \underset{n \rightarrow \infty}{\stackrel{d}{\longrightarrow}} \omega^{2} K_{c}^{2}(1) / 2-\sigma^{2} / 2-\omega^{2} \int_{0}^{1} c f(r) K_{c}^{2}(r) d r .
$$

Stochastic differentiation of $K_{c}^{2}(r)$ gives $K_{c}^{2}(r)=r+2 \int_{0}^{r} c f(r) K_{c}^{2}(r) d r+2 \int_{0}^{r} K_{c}(r) d W(r)$, from which we deduce that $n^{-1} \sum_{t=0}^{n} X_{t-1} u_{t} \underset{n \rightarrow \infty}{\stackrel{d}{\longrightarrow}} \omega^{2} \int_{0}^{1} K_{c}(r) d W(r)+\lambda$, where $\lambda=$ $\sum_{h=1}^{\infty} \mathbf{E} u_{o} u_{h}=\frac{1}{2}\left(\omega^{2}-\sigma^{2}\right), B(r)=B^{a}(r) \times \mathbf{1}\left\{0 \leq r \leq r_{1}\right\}+B(r) \times \mathbf{1}\left\{r_{1}<r \leq r_{2}\right\}+B^{b}(r) \times$ $\mathbf{1}\left\{r_{2}<r \leq 1\right\}$. Thus, as $n \rightarrow \infty$ we find that

$$
\begin{aligned}
& n\left(\hat{\theta}_{O L S}-1-\frac{\int_{r_{1}}^{r_{2}} c f(r) F_{c}^{2}(r) d r}{n \int_{0}^{1} F_{c}(r)^{2} d r}\right) \underset{n \rightarrow \infty}{\longrightarrow} \frac{\int_{0}^{1} F_{c}(r) d B(r)+\lambda}{\int_{0}^{1} F_{c}(r)^{2} d r}, \\
& n\left(\hat{\theta}_{O L S}-1\right) \underset{n \rightarrow \infty}{\longrightarrow} \frac{\int_{0}^{1} F_{c}(r) d B(r)+\lambda+\int_{r_{1}}^{r_{2}} c f(r) F_{c}^{2}(r) d r}{\int_{0}^{1} F_{c}(r)^{2} d r} .
\end{aligned}
$$

The limits of $\int_{0}^{1} F_{c}(r) d B(r)+\lambda$ and $\int_{0}^{1} F_{c}(r)^{2} d r$ as $c \rightarrow-\infty$ are straightforward and we find the following centered limit theory in the boundary asymptotics

$$
\begin{aligned}
& n\left(\hat{\theta}_{O L S}-1-\frac{\int_{r_{1}}^{r_{2}} c f(r) F_{c}^{2}(r) d r}{n \int_{0}^{1} F_{c}(r)^{2} d r}\right) \\
& \underset{(-c, n)_{\text {seq. }} \rightarrow \infty}{\longrightarrow} \frac{\int_{0}^{r_{1}} B^{a}(r) d B^{a}(r)+\int_{r_{2}}^{1} B^{b}(r) d B^{b}(r)+\lambda}{\int_{0}^{r_{1}} B^{a}(r)^{2} d r+\int_{r_{2}}^{1} B^{b}(r)^{2} d r} .
\end{aligned}
$$

Note that the centering of the limit theory for $\hat{\theta}$ in 33 is stochastic and involves the weighted quantity $\int_{r_{1}}^{r_{2}} c f(r) F_{c}^{2}(r) d r$ whose limit behavior is complicated. We proceed to calculate this limit to develop a non random centering in place of (33).

$$
\begin{aligned}
c \int_{r_{1}}^{r_{2}} f(r) F_{c}^{2}(r) d r & =\omega^{2} c \int_{r_{1}}^{r_{2}} f(r)\left(e^{c \int_{r_{1}}^{r} f(a) d a} W^{a}\left(r_{1}\right)+\int_{r_{1}}^{r} e^{c \int_{s}^{r} f(a) d a} d W(s)\right)^{2} d r \\
& =\omega^{2} c W^{a}\left(r_{1}\right)^{2} \int_{r_{1}}^{r_{2}} f(r) e^{2 c \int_{r_{1}}^{r} f(a) d a} d r \\
& +2 \omega^{2} c W^{a}\left(r_{1}\right) \int_{r_{1}}^{r_{2}} f(r) e^{c \int_{r_{1}}^{r} f(a) d a} \int_{r_{1}}^{r} e^{c \int_{s}^{r} f(a) d a} d W(s) d r \\
& +\omega^{2} c \int_{r_{1}}^{r_{2}} f(r)\left(\int_{r_{1}}^{r} e^{c \int_{s}^{r} f(a) d a} d W(s)\right)^{2} d r .
\end{aligned}
$$


We evaluate the limit of each of the three terms in Eq. (34). First, denoting $\partial_{r}:=\frac{\partial}{\partial r}$,

$$
\begin{aligned}
& \omega^{2} c W^{a}\left(r_{1}\right)^{2} \int_{r_{1}}^{r_{2}} f(r) e^{2 c \int_{r_{1}}^{r} f(a) d a} d r=\omega^{2} c W^{a}\left(r_{1}\right)^{2} \int_{r_{1}}^{r_{2}} \partial_{r}\left(\frac{1}{2 c} e^{2 c \int_{r_{1}}^{r} f(a) d a}\right) d r \\
& =\omega^{2} W^{a}\left(r_{1}\right)^{2} \frac{1}{2}\left(e^{2 c \int_{r_{1}}^{r_{2}} f(a) d a}-1\right) \underset{c \rightarrow-\infty}{\stackrel{d}{\longrightarrow}}-\frac{1}{2} B^{a}\left(r_{1}\right)^{2} .
\end{aligned}
$$

The second term has mean

$$
\mathbb{E}\left[2 c W^{a}\left(r_{1}\right) \int_{r_{1}}^{r_{2}} f(r) e^{c \int_{r_{1}}^{r} f(a) d a} \int_{r_{1}}^{r} e^{c \int_{s}^{r} f(a) d a} d W(s) d r\right]=0,
$$

as $W^{a}$ and $W$ are independent. We now show that the variance of this term converges to zero as $c \rightarrow-\infty$, so that the term tends in probability to zero. We have

$$
\begin{aligned}
& \mathbb{E}\left[2 c W^{a}\left(r_{1}\right) \int_{r_{1}}^{r_{2}} f(r) e^{c \int_{r_{1}}^{r} f(a) d a} \int_{r_{1}}^{r} e^{c \int_{s}^{r} f(a) d a} d W(s) d r\right]^{2} \\
& =4 r_{1} c^{2} \mathbb{E}\left[\int_{r_{1}}^{r_{2}} f(r) e^{c \int_{r_{1}}^{r} f(a) d a} \int_{r_{1}}^{r} e^{c \int_{s}^{r} f(a) d a} d W(s) d r\right]^{2} \\
& =4 r_{1} c^{2} \mathbb{E}\left[\int_{r_{1}}^{r_{2}} \int_{s}^{r_{2}} f(r) e^{c \int_{r_{1}}^{r} f(a) d a} e^{c \int_{s}^{r} f(a) d a} d r d W(s)\right]^{2} \\
& =4 r_{1} c^{2} \int_{r_{1}}^{r_{2}}\left(\int_{s}^{r_{2}} f(r) e^{c \int_{r_{1}}^{r} f(a) d a} e^{c \int_{s}^{r} f(a) d a} d r\right)^{2} d s \\
& =4 r_{1} c^{2} \int_{r_{1}}^{r_{2}}\left(\frac{1}{2 c}\left(e^{c \int_{r_{1}}^{r_{2}} f(a) d a+c \int_{s}^{r_{2}} f(a) d a}-e^{c \int_{r_{1}}^{s} f(a) d a}\right)\right)^{2} d s \\
& =r_{1} \int_{r_{1}}^{r_{2}}\left(e^{2 c \int_{r_{1}}^{r_{2}} f(a) d a+2 c \int_{s}^{r_{2}} f(a) d a}+e^{2 c \int_{r_{1}}^{s} f(a) d a}-2 e^{2 c \int_{r_{1}}^{r_{2}} f(a) d a}\right) d r \underset{c \rightarrow-\infty}{\longrightarrow} 0
\end{aligned}
$$

and hence

$$
2 \omega^{2} c W^{a}\left(r_{1}\right) \int_{r_{1}}^{r_{2}} f(r) e^{c \int_{r_{1}}^{r} f(a) d a} \int_{r_{1}}^{r} e^{c \int_{s}^{r} f(a) d a} d W(s) d r \underset{c \rightarrow-\infty}{\stackrel{p}{\longrightarrow}} 0
$$

We are left with the third term. As with the second term, we show that the variance converges to zero, and thus the whole term converges to the limit of its expectation. We 
first calculate this limit as follows

$$
\begin{aligned}
& \mathbb{E}\left[\omega^{2} c \int_{r_{1}}^{r_{2}} f(r)\left(\int_{r_{1}}^{r} e^{c \int_{s}^{r} f(a) d a} d W(s)\right)^{2} d r\right] \\
& =\omega^{2} c \int_{r_{1}}^{r_{2}} f(r) \mathbb{E}\left[\int_{r_{1}}^{r} e^{c \int_{s}^{r} f(a) d a} d W(s)\right]^{2} d r=\omega^{2} c \int_{r_{1}}^{r_{2}} f(r) \int_{r_{1}}^{r} e^{2 c \int_{s}^{r} f(a) d a} d s d r \\
& =\omega^{2} c \int_{r_{1}}^{r_{2}} \int_{s}^{r_{2}} f(r) e^{2 c \int_{s}^{r} f(a) d a} d r d s=\frac{\omega^{2}}{2} \int_{r_{1}}^{r_{2}}\left(e^{2 c \int_{s}^{r_{2}} f(a) d a}-1\right) d s \\
& =\frac{\omega^{2}}{2} \int_{r_{1}}^{r_{2}} e^{2 c \int_{s}^{r_{2}} f(a) d a} d s-\frac{\omega^{2}}{2}\left(r_{2}-r_{1}\right) \underset{c \rightarrow-\infty}{\longrightarrow}-\frac{\omega^{2}}{2}\left(r_{2}-r_{1}\right) .
\end{aligned}
$$

We proceed to show that the variance of the third term goes to zero. In what follows, the variance of a random variable $\xi$ is denoted by $\mathbb{V} \xi$ and the covariance between $\xi_{1}$ and $\xi_{2}$ is denoted by $\operatorname{cov}\left(\xi_{1}, \xi_{2}\right)$. We use the fact that for a stochastic process $\xi_{t}$ with finite second moments we have

$$
\begin{aligned}
\mathbb{V} \int_{r_{1}}^{r_{2}} \xi_{t} d t & =\mathbb{E}\left(\int_{r_{1}}^{r_{2}} \xi_{t} d t\right)^{2}-\left(\mathbb{E} \int_{r_{1}}^{r_{2}} \xi_{t} d t\right)^{2} \\
& =\mathbb{E}\left(\int_{r_{1}}^{r_{2}} \xi_{t} d t \int_{r_{1}}^{r_{2}} \xi_{s} d s\right)-\mathbb{E} \int_{r_{1}}^{r_{2}} \xi_{t} d t \mathbb{E} \int_{r_{1}}^{r_{2}} \xi_{s} d s \\
& =\int_{r_{1}}^{r_{2}} \int_{r_{1}}^{r_{2}} \mathbb{E}\left(\xi_{t} \xi_{s}\right) d s d t-\int_{r_{1}}^{r_{2}} \int_{r_{1}}^{r_{2}}\left(\mathbb{E} \xi_{t}\right)\left(\mathbb{E} \xi_{s}\right) d s d t=\int_{r_{1}}^{r_{2}} \int_{r_{1}}^{r_{2}} \operatorname{cov}\left(\xi_{t}, \xi_{s}\right) d s d t .
\end{aligned}
$$

Therefore,

$$
\begin{aligned}
& \mathbb{V}\left[c \int_{r_{1}}^{r_{2}} f(r)\left(\int_{r_{1}}^{r} e^{c \int_{s}^{r} f(a) d a} d W(s)\right)^{2} d r\right] \\
& =c^{2} \int_{r_{1}}^{r_{2}} \int_{r_{1}}^{r_{2}} f(r) f\left(r^{\prime}\right) \operatorname{cov}\left\{\left(\int_{r_{1}}^{r} e^{c \int_{s}^{r} f(a) d a} d W(s)\right)^{2},\left(\int_{r_{1}}^{r^{\prime}} e^{c \int_{s}^{r^{\prime}} f(a) d a} d W(s)\right)^{2}\right\} d r^{\prime} d r .
\end{aligned}
$$

For this calculation, we need the covariance between two squared normal variables with zero mean. By Isselis's theorem, if $\xi_{r}, \xi_{r^{\prime}}$ are normally distributed (possible dependent) with zero mean, then

$$
\operatorname{cov}\left(\xi_{r}^{2}, \xi_{r^{\prime}}^{2}\right) \equiv \mathbb{E} \xi_{r}^{2} \xi_{r^{\prime}}^{2}-\mathbb{E} \xi_{r}^{2} \mathbb{E} \xi_{r^{\prime}}^{2}=2\left(\mathbb{E} \xi_{r} \xi_{r^{\prime}}\right)^{2}
$$


Thus, we can rewrite Eq. (39) as

$$
\begin{aligned}
& \mathbb{V}\left[c \int_{r_{1}}^{r_{2}} f(r)\left(\int_{r_{1}}^{r} e^{c \int_{s}^{r} f(a) d a} d W(s)\right)^{2} d r\right] \\
& =2 c^{2} \int_{r_{1}}^{r_{2}} \int_{r_{1}}^{r_{2}} f(r) f\left(r^{\prime}\right)\left(\mathbb{E} \int_{r_{1}}^{r} e^{c \int_{s}^{r} f(a) d a} d W(s) \int_{r_{1}}^{r^{\prime}} e^{c \int_{s}^{r^{\prime}} f(a) d a} d W(s)\right)^{2} d r^{\prime} d r \\
& =2 c^{2} \int_{r_{1}}^{r_{2}} \int_{r_{1}}^{r_{2}} f(r) f\left(r^{\prime}\right)\left(\int_{r_{1}}^{\min \left(r, r^{\prime}\right)} e^{c \int_{s}^{r} f(a) d a} e^{c \int_{s}^{r^{\prime}} f(a) d a} d s\right)^{2} d r^{\prime} d r \\
& =4 c^{2} \int_{r_{1}}^{r_{2}} \int_{r_{1}}^{r} f(r) f\left(r^{\prime}\right) e^{2 c \int_{r^{\prime}}^{r} f(a) d a}\left(\int_{r_{1}}^{r^{\prime}} e^{2 c \int_{s}^{r^{\prime}} f(a) d a} d s\right)^{2} d r^{\prime} d r \\
& =4 c^{2} \int_{r_{1}}^{r_{2}} \int_{r_{1}}^{r} \int_{r_{1}}^{r^{\prime}} \int_{r_{1}}^{r^{\prime}} f(r) f\left(r^{\prime}\right) e^{2 c \int_{r^{\prime}}^{r} f(a) d a} e^{2 c \int_{s}^{r^{\prime}} f(a) d a} e^{2 c \int_{s^{\prime}}^{r^{\prime}} f(a) d a} d s^{\prime} d s d r^{\prime} d r \\
& =4 c^{2} \int_{r_{1}}^{r_{2}} \int_{s}^{r_{2}} \int_{r^{\prime}}^{r_{2}}\left(\int_{r_{1}}^{r^{\prime}} e^{2 c \int_{s^{\prime}}^{r^{\prime}} f(a) d a} d s^{\prime}\right) f(r) e^{2 c \int_{r^{\prime}}^{r} f(a) d a} d r f\left(r^{\prime}\right) e^{2 c \int_{s}^{r^{\prime}} f(a) d a} d r^{\prime} d s \\
& =2 c \int_{r_{1}}^{r_{2}} \int_{s}^{r_{2}}\left(\int_{r_{1}}^{r^{\prime}} e^{2 c \int_{s^{\prime}}^{r^{\prime}} f(a) d a} d s^{\prime}\right)\left(e^{2 c \int_{r^{\prime}}^{r_{2}} f(a) d a}-1\right) f\left(r^{\prime}\right) e^{2 c \int_{s}^{r^{\prime}} f(a) d a} d r^{\prime} d s \\
& =2 c \int_{r_{1}}^{r_{2}} \int_{\max \left(s, s^{\prime}\right)}^{2 c \int_{s^{\prime}}^{r^{\prime}} f(a) d a}\left(e^{2 c \int_{r^{\prime}}^{r_{2}} f(a) d a}-1\right) f\left(r^{\prime}\right) e^{2 c \int_{s}^{r^{\prime}} f(a) d a} d r^{\prime} d s^{\prime} d s, \\
& =
\end{aligned}
$$

with

$$
\begin{aligned}
& 2 c \int_{r_{1}}^{r_{2}} \int_{r_{1}}^{r_{2}} \int_{\max \left(s, s^{\prime}\right)}^{r_{2}} e^{2 c \int_{s^{\prime}}^{r^{\prime}} f(a) d a} f\left(r^{\prime}\right) e^{2 c \int_{r^{\prime}}^{r_{2}} f(a) d a} e^{2 c \int_{s}^{r^{\prime}} f(a) d a} d r^{\prime} d s^{\prime} d s \\
& =\int_{r_{1}}^{r_{2}} \int_{r_{1}}^{r_{2}} e^{2 c \int_{s}^{r_{2}} f(a) d a}\left(e^{2 c \int_{s^{\prime}}^{r_{2}} f(a) d a}-e^{2 c \int_{s^{\prime}}^{\max \left(s, s^{\prime}\right)} f(a) d a}\right) d s^{\prime} d s \\
& =\left(\int_{r_{1}}^{r_{2}} e^{2 c \int_{s}^{r_{2}} f(a) d a} d s\right)^{2}-\int_{r_{1}}^{r_{2}} e^{2 c \int_{s}^{r_{2}} f(a) d a}\left(\int_{r_{1}}^{s} e^{2 c \int_{s^{\prime}}^{s} f(a) d a} d s^{\prime}+\int_{s}^{r_{2}} d s^{\prime}\right) d s \\
& =\left(\int_{r_{1}}^{r_{2}} e^{2 c \int_{s}^{r_{2}} f(a) d a} d s\right)^{2}-\int_{r_{1}}^{r_{2}} \int_{r_{1}}^{s} e^{2 c \int_{s^{\prime}}^{r_{2}} f(a) d a} d s^{\prime} d s-\int_{r_{1}}^{r_{2}}\left(r_{2}-s\right) e^{2 c \int_{s}^{r_{2}} f(a) d a} d s,
\end{aligned}
$$


and

$$
\begin{aligned}
& 2 c \int_{r_{1}}^{r_{2}} \int_{r_{1}}^{r_{2}} \int_{\max \left(s, s^{\prime}\right)}^{r_{2}} e^{2 c \int_{s^{\prime}}^{r^{\prime}} f(a) d a} f\left(r^{\prime}\right) e^{2 c \int_{s}^{r^{\prime}} f(a) d a} d r^{\prime} d s^{\prime} d s \\
& =\frac{1}{2} \int_{r_{1}}^{r_{2}} \int_{r_{1}}^{r_{2}}\left(e^{2 c \int_{s^{\prime}}^{r_{2}} f(a) d a+2 c \int_{s}^{r_{2}} f(a) d a}-e^{2 c \int_{s^{\prime}}^{\max \left(s, s^{\prime}\right)} f(a) d a+2 c \int_{s}^{\max \left(s, s^{\prime}\right)} f(a) d a}\right) d s^{\prime} d s \\
& =\frac{1}{2}\left(\int_{r_{1}}^{r_{2}} e^{2 c \int_{s}^{r_{2}} f(a) d a} d s\right)^{2}-\int_{r_{1}}^{r_{2}} \int_{r_{1}}^{s} e^{2 c \int_{s^{\prime}}^{s} f(a) d a} d s^{\prime} d s .
\end{aligned}
$$

Using (42) and (43) in (41) gives

$$
\begin{aligned}
& \mathbb{V}\left[c \int_{r_{1}}^{r_{2}} f(r)\left(\int_{r_{1}}^{r} e^{c \int_{s}^{r} f(a) d a} d W(s)\right)^{2} d r\right] \\
& =\frac{1}{2}\left(\int_{r_{1}}^{r_{2}} e^{2 c \int_{s}^{r_{2}} f(a) d a} d s\right)^{2}-\int_{r_{1}}^{r_{2}} \int_{r_{1}}^{s} e^{2 c \int_{s^{\prime}}^{r_{2}} f(a) d a} d s^{\prime} d s-\int_{r_{1}}^{r_{2}}\left(r_{2}-s\right) e^{2 c \int_{s}^{r_{2}} f(a) d a} d s \\
& +\int_{r_{1}}^{r_{2}} \int_{r_{1}}^{s} e^{2 c \int_{s^{\prime}}^{s} f(a) d a} d s^{\prime} d s \underset{c \rightarrow-\infty}{\longrightarrow} 0,
\end{aligned}
$$

as each term goes to zero. It follows that

$$
\omega^{2} c \int_{r_{1}}^{r_{2}} f(r)\left(\int_{r_{1}}^{r} e^{c \int_{s}^{r} f(a) d a} d W(s)\right)^{2} d r \underset{c \rightarrow-\infty}{\stackrel{p}{\longrightarrow}}-\frac{\omega^{2}}{2}\left(r_{2}-r_{1}\right) .
$$

and using 35, (37), and (45) in (34), we obtain

$$
\int_{r_{1}}^{r_{2}} c f(r) F_{c}^{2}(r) d r \underset{c \rightarrow-\infty}{\stackrel{d}{\longrightarrow}}-\frac{1}{2}\left(\omega^{2}\left(r_{2}-r_{1}\right)+B^{a}\left(r_{1}\right)^{2}\right) .
$$

Finally, taking the limit as $c \rightarrow-\infty$ in (32) leads to a form of segmented unit root limit distribution as follows

$$
\begin{aligned}
& \frac{\int_{0}^{1} F_{c}(r) d B(r)+\lambda+\int_{r_{1}}^{r_{2}} c f(r) F_{c}^{2}(r) d r}{\int_{0}^{1} F_{c}(r)^{2} d r} \\
& \underset{c \rightarrow-\infty}{\longrightarrow} \frac{\int_{0}^{r_{1}} B^{a}(r) d B^{a}(r)+\int_{r_{2}}^{1} B^{b}(r) d B^{b}(r)+\lambda-\frac{1}{2}\left(\omega^{2}\left(r_{2}-r_{1}\right)+B^{a}\left(r_{1}\right)^{2}\right)}{\int_{0}^{r_{1}} B^{a}(r)^{2} d r+\int_{r_{2}}^{1} B^{b}(r)^{2} d r} \\
& =\frac{\int_{r_{2}}^{1} B^{b}(r) d B^{b}(r)+\lambda-\frac{\omega^{2} r_{2}}{2}}{\int_{0}^{r_{1}} B^{a}(r)^{2} d r+\int_{r_{2}}^{1} B^{b}(r)^{2} d r} .
\end{aligned}
$$

Using these results and working in a similar way, we can derive the boundary limit behavior of the unit root $t$ statistic associated with $\hat{\theta}_{O L S}$, i.e.,

$$
t_{\hat{\theta}_{O L S}}=\frac{\hat{\theta}_{O L S}-1}{\hat{s}_{\hat{\theta}_{O L S}}}:=\frac{\sqrt{\sum_{t=1}^{n} X_{t-1}^{2}}\left(\hat{\theta}_{O L S}-1\right)}{\sqrt{\frac{1}{n} \sum_{t=1}^{n}\left(X_{t}-\hat{\theta}_{O L S} X_{t-1}\right)^{2}}} .
$$


Since $\hat{\theta}_{O L S}=1+O_{p}\left(n^{-1}\right)=1+c f(t / n) / n+O_{p}\left(n^{-1}\right)$, it follows that

$$
n^{-1} \sum_{t=1}^{n}\left(X_{t}-\hat{\theta}_{O L S} X_{t-1}\right)^{2} \underset{n \rightarrow \infty}{\stackrel{p}{\longrightarrow}} \sigma^{2}
$$

and we have already shown that

$$
\begin{aligned}
& n\left(\hat{\theta}_{O L S}-1\right) \underset{n \rightarrow \infty}{\stackrel{d}{\longrightarrow}} \frac{\int_{0}^{1} F_{c}(r) d B(r)+\lambda+\int_{r_{1}}^{r_{2}} c f(r) F_{c}^{2}(r) d r}{\int_{0}^{1} F_{c}(r)^{2} d r}, \\
& \int_{0}^{1} F_{c}(r) d B(r)+\lambda \underset{c \rightarrow-\infty}{\stackrel{d}{\longrightarrow}} \int_{0}^{r_{1}} B^{a}(r) d B^{a}(r)+\int_{r_{2}}^{1} B^{b}(r) d B^{b}(r)+\lambda, \\
& \int_{r_{1}}^{r_{2}} c f(r) F_{c}^{2}(r) d r \underset{c \rightarrow-\infty}{\stackrel{d}{\longrightarrow}}-\frac{1}{2}\left(\omega^{2}\left(r_{2}-r_{1}\right)+B^{a}\left(r_{1}\right)^{2}\right) \\
& \int_{0}^{1} F_{c}(r)^{2} d r \underset{c \rightarrow-\infty}{\stackrel{d}{\longrightarrow}} \int_{0}^{r_{1}} B^{a}(r)^{2} d r+\int_{r_{2}}^{1} B^{b}(r)^{2} d r,
\end{aligned}
$$

Combining these limits yields the desired result

$$
\begin{aligned}
& t_{\hat{\theta}_{O L S}} \underset{(-c, n)_{s e q} \rightarrow \infty}{\longrightarrow} \frac{\int_{0}^{r_{1}} B^{a}(r) d B^{a}(r)+\int_{r_{2}}^{1} B^{b}(r) d B^{b}(r)+\lambda-\frac{1}{2}\left(\omega^{2}\left(r_{2}-r_{1}\right)+B^{a}\left(r_{1}\right)^{2}\right)}{\sigma\left(\int_{0}^{r_{1}} B^{a}(r)^{2} d r+\int_{r_{2}}^{1} B^{b}(r)^{2} d r\right)^{1 / 2}} \\
& =\frac{\int_{r_{2}}^{1} B^{b}(r) d B^{b}(r)+\lambda-\frac{\omega^{2} r_{2}}{2}}{\sigma\left(\int_{0}^{r_{1}} B^{a}(r)^{2} d r+\int_{r_{2}}^{1} B^{b}(r)^{2} d r\right)^{1 / 2}} .
\end{aligned}
$$

\section{Proof of Theorem 2,}

Proof. By virtue of Eq. (2), the limit process for $X_{t}$ as $n \rightarrow \infty$ has the segmented form

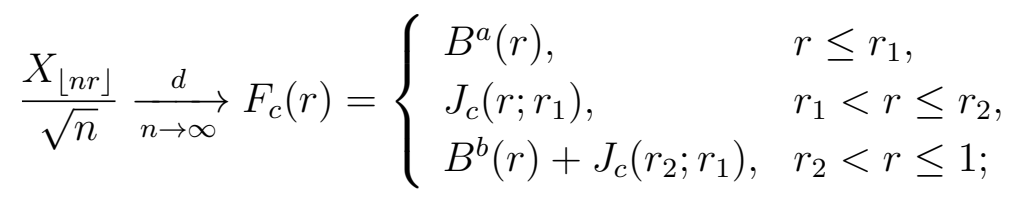

with $B^{a} \perp B^{b}$ and

$$
J_{c}\left(r ; r_{1}\right)=\int_{0}^{r} e^{c \int_{s}^{r} f(a) d a} d B(s)=e^{c \int_{r_{1}}^{r} f(a) d a} B^{a}\left(r_{1}\right)+\int_{r_{1}}^{r} e^{c \int_{s}^{r} f(a) d a} d B(s) .
$$


So

$$
\begin{aligned}
e^{-c \int_{r_{1}}^{r} f(a) d a} J_{c}\left(r ; r_{1}\right) & =B^{a}\left(r_{1}\right)+\int_{r_{1}}^{r} e^{-c \int_{r_{1}}^{r} f(a) d a} e^{c \int_{s}^{r} f(a) d a} d B(s) \\
& =B^{a}\left(r_{1}\right)+\int_{r_{1}}^{r} e^{-c \int_{r_{1}}^{s} f(a) d a} d B(s)=B^{a}\left(r_{1}\right)+o_{p}(1) .
\end{aligned}
$$

Thus, for all $r$,

$$
e^{-c \int_{r_{1}}^{r_{2}} f(a) d a} B(r)=e^{-c \int_{r_{1}}^{r_{2}} f(a) d a} \times O_{p}(1) \underset{c \rightarrow \infty}{\stackrel{d}{\longrightarrow}} 0,
$$

and, for all $r<r_{2}$

$$
e^{-c \int_{r_{1}}^{r_{2}} f(a) d a} J_{c}\left(r ; r_{1}\right)=e^{-c \int_{r}^{r_{2}} f(a) d a}\left(B^{a}\left(r_{1}\right)+o_{p}(1)\right) \underset{c \rightarrow \infty}{\stackrel{d}{\longrightarrow}} 0,
$$

while

$$
e^{-c \int_{r_{1}}^{r_{2}} f(a) d a} J_{c}\left(r_{2} ; r_{1}\right) \underset{c \rightarrow \infty}{\stackrel{d}{\longrightarrow}} B^{a}\left(r_{1}\right)
$$

Thus $e^{-c \int_{r_{1}}^{r_{2}} f(a) d a} \frac{X_{\lfloor n r\rfloor}}{\sqrt{n}} \underset{(c, n)_{s e q} \rightarrow \infty}{\longrightarrow} B^{a}\left(r_{1}\right) \times \mathbf{1}\left\{r_{2} \leq r \leq 1\right\}$.

By the same argument as in the proof of Theorem 1.

$$
\begin{aligned}
n\left(\hat{\theta}_{O L S}-1\right)= & \frac{\frac{1}{n} \sum_{t=0}^{n} X_{t-1} u_{t}}{\frac{1}{n^{2}} \sum_{t=0}^{n} X_{t-1}^{2}}+\frac{\frac{1}{n} \sum_{t} \frac{c f(t / n)}{n} X_{t-1}^{2}}{\frac{1}{n^{2}} \sum_{t} X_{t-1}^{2}} \\
& \underset{n \rightarrow \infty}{\stackrel{d}{\longrightarrow}} \frac{\int_{0}^{1} F_{c}(r) d B(r)+\lambda+\int_{r_{1}}^{r_{2}} c f(r) F_{c}(r)^{2} d r}{\int_{0}^{1} F_{c}(r)^{2} d r},
\end{aligned}
$$

so that

$$
n\left(\hat{\theta}_{O L S}-1-\frac{\int_{r_{1}}^{r_{2}} c f(r) F_{c}(r)^{2} d r}{n \int_{0}^{1} F_{c}(r)^{2} d r}\right) \underset{n \rightarrow \infty}{\stackrel{d}{\longrightarrow}} \frac{\int_{0}^{1} F_{c}(r) d B(r)+\lambda}{\int_{0}^{1} F_{c}(r)^{2} d r} .
$$

Using Eq. 48, we can calculate the limit of the denominator $\int_{0}^{1} F_{c}(r)^{2} d r$ as $c$ goes to infinity. First,

$$
\int_{0}^{r_{1}} F_{c}(r)^{2} d r=\int_{0}^{r_{1}}\left(B^{a}(r)\right)^{2} d r=O_{p}(1)
$$


Then,

(50)

$$
\begin{aligned}
\int_{r_{1}}^{r_{2}} F_{c}(r)^{2} d r & =\int_{r_{1}}^{r_{2}} J_{c}\left(r ; r_{1}\right)^{2} d r=\int_{r_{1}}^{r_{2}}\left[e^{c \int_{r_{1}}^{r} f(a) d a} B^{a}\left(r_{1}\right)+\int_{r_{1}}^{r} e^{c \int_{s}^{r} f(a) d a} d B(s)\right]^{2} d r \\
& =\int_{r_{1}}^{r_{2}} e^{2 c \int_{r_{1}}^{r} f(a) d a}\left[B^{a}\left(r_{1}\right)+\int_{r_{1}}^{r} e^{-c \int_{r_{1}}^{s} f(a) d a} d B(s)\right]^{2} d r \\
& =\int_{r_{1}}^{r_{2}} e^{2 c \int_{r_{1}}^{r} f(a) d a}\left(B^{a}\left(r_{1}\right)+o_{p}(1)\right)^{2} d r \\
& =\left(B^{a}\left(r_{1}\right)\right)^{2} \int_{r_{1}}^{r_{2}} e^{2 c \int_{r_{1}}^{r} f(a) d a} d r+o_{p}(1) \int_{r_{1}}^{r_{2}} e^{2 c \int_{r_{1}}^{r} f(a) d a} d r .
\end{aligned}
$$

Finally,

$$
\begin{aligned}
\int_{r_{2}}^{1} F_{c}(r)^{2} d r & =\int_{r_{2}}^{1}\left(B^{b}(r)+J_{c}\left(r_{2} ; r_{1}\right)\right)^{2} d r \\
& =\int_{r_{2}}^{1} B^{b}(r)^{2} d r+2 \int_{r_{2}}^{1} B^{b}(r) J_{c}\left(r_{2} ; r_{1}\right) d r+\int_{r_{2}}^{1} J_{c}\left(r_{2} ; r_{1}\right)^{2} d r .
\end{aligned}
$$

Hence, using Eq. 47), we have

$$
\begin{aligned}
e^{-2 c \int_{r_{1}}^{r_{2}} f(a) d a} \int_{r_{2}}^{1} F_{c}(r)^{2} d r & =o_{p}(1)+2 B^{a}\left(r_{1}\right) e^{-c \int_{r_{1}}^{r_{2}} f(a) d a} \int_{r_{2}}^{1} B^{b}(r) d r \\
& +\left(1-r_{2}\right)\left(B^{a}\left(r_{1}\right)\right)^{2}=\left(1-r_{2}\right)\left(B^{a}\left(r_{1}\right)\right)^{2}+o_{p}(1) .
\end{aligned}
$$

Combining Eq. 49, Eq. 50, and Eq. 51 then yields

$$
\begin{aligned}
e^{-2 c \int_{r_{1}}^{r_{2}} f(a) d a} \int_{0}^{1} F_{c}(r)^{2} d r & =o_{p}(1)+\left(B^{a}\left(r_{1}\right)\right)^{2} \int_{r_{1}}^{r_{2}} e^{-2 c \int_{r}^{r_{2}} f(a) d a} d r \\
& +o_{p}(1) \int_{r_{1}}^{r_{2}} e^{-2 c \int_{r}^{r_{2}} f(a) d a} d r+\left(1-r_{2}\right)\left(B^{a}\left(r_{1}\right)\right)^{2} \\
& =\left(1-r_{2}\right)\left(B^{a}\left(r_{1}\right)\right)^{2}+o_{p}(1) .
\end{aligned}
$$

In a similar way, we can analyze the first part of the numerator, $\int_{0}^{1} F_{c}(r) d B(r)+\lambda$. Note that

$$
\begin{aligned}
\int_{0}^{1} F_{c}(r) d B(r) & =\int_{0}^{r_{1}} B^{a}(r) d B^{a}(r)+\int_{r_{1}}^{r_{2}} J_{c}\left(r ; r_{1}\right) d B(r) \\
& +\int_{r_{2}}^{1}\left(B^{b}(r)+J_{c}\left(r_{2} ; r_{1}\right)\right) d B^{b}(r) \\
& =\int_{0}^{r_{1}} B^{a}(r) d B^{a}(r)+\int_{r_{2}}^{1} B^{b}(r) d B^{b}(r) \\
& +\int_{r_{1}}^{r_{2}} J_{c}\left(r ; r_{1}\right) d B(r)+J_{c}\left(r_{2} ; r_{1}\right)\left(B^{b}(1)-B^{b}\left(r_{2}\right)\right) .
\end{aligned}
$$


Thus,

$$
\begin{aligned}
& e^{-c \int_{r_{1}}^{r_{2}} f(a) d a}\left[\int_{0}^{1} F_{c}(r) d B(r)+\lambda\right]=o_{p}(1)+B^{a}\left(r_{1}\right)\left(B^{b}(1)-B^{b}\left(r_{2}\right)\right) \\
& +\int_{r_{1}}^{r_{2}} e^{-c \int_{r}^{r_{2}} f(a) d a}\left(B^{a}\left(r_{1}\right)+o_{p}(1)\right) d B(r) \\
& =B^{a}\left(r_{1}\right)\left(B^{b}(1)-B^{b}\left(r_{2}\right)\right)+o_{p}(1) .
\end{aligned}
$$

Combining Eq. 52 and Eq. 53, we obtain

$$
\begin{aligned}
\frac{e^{-c \int_{r_{1}}^{r_{2}} f(a) d a}\left[\int_{0}^{1} F_{c}(r) d B(r)+\lambda\right]}{e^{-2 c \int_{r_{1}}^{r_{2}} f(a) d a} \int_{0}^{1} F_{c}(r)^{2} d r} & \frac{d}{c \rightarrow \infty} \frac{B^{a}\left(r_{1}\right)\left(B^{b}(1)-B^{b}\left(r_{2}\right)\right)}{\left(1-r_{2}\right)\left(B^{a}\left(r_{1}\right)\right)^{2}} \\
& =\frac{B^{b}(1)-B^{b}\left(r_{2}\right)}{\left(1-r_{2}\right) B^{a}\left(r_{1}\right)} \\
& =\frac{\mathcal{N}\left(0,1-r_{2}\right)}{\left(1-r_{2}\right) \mathcal{N}\left(0, r_{1}\right)}=\frac{1}{\sqrt{r_{1}\left(1-r_{2}\right)}} \mathcal{C} .
\end{aligned}
$$

Thus

$$
e^{c \int_{r_{1}}^{r_{2}} f(a) d a} n\left(\hat{\theta}-1-\frac{\int_{r_{1}}^{r_{2}} c f(r) F_{c}(r)^{2} d r}{n \int_{0}^{1} F_{c}(r)^{2} d r}\right) \underset{d}{\stackrel{d}{(c, n)_{s e q} \rightarrow \infty}} \frac{1}{\sqrt{r_{1}\left(1-r_{2}\right)}} \mathcal{C}
$$

leading to a scaled Cauchy distribution in the limit.

We are left to analyze the second part of the numerator, $\int_{r_{1}}^{r_{2}} c f(r) F_{c}(r)^{2} d r$. The analysis is almost identical to the case $c \rightarrow-\infty$ studied in the proof of Theorem 1. By Eq. (34),

$$
\begin{aligned}
c \int_{r_{1}}^{r_{2}} f(r) F_{c}^{2}(r) d r & =\omega^{2} c W^{a}\left(r_{1}\right)^{2} \int_{r_{1}}^{r_{2}} f(r) e^{2 c \int_{r_{1}}^{r} f(a) d a} d r \\
& +2 \omega^{2} c W^{a}\left(r_{1}\right) \int_{r_{1}}^{r_{2}} f(r) e^{c \int_{r_{1}}^{r} f(a) d a} \int_{r_{1}}^{r} e^{c \int_{s}^{r} f(a) d a} d W(s) d r \\
& +\omega^{2} c \int_{r_{1}}^{r_{2}} f(r)\left(\int_{r_{1}}^{r} e^{c \int_{s}^{r} f(a) d a} d W(s)\right)^{2} d r .
\end{aligned}
$$

By Eq. 35, for the first term we have

$$
\begin{aligned}
& e^{-2 c \int_{r_{1}}^{r_{2}} f(a) d a} \omega^{2} c W^{a}\left(r_{1}\right)^{2} \int_{r_{1}}^{r_{2}} f(r) e^{2 c \int_{r_{1}}^{r} f(a) d a} d r \\
& =\omega^{2} W^{a}\left(r_{1}\right)^{2} \frac{1}{2}\left(1-e^{-2 c \int_{r_{1}}^{r_{2}} f(a) d a} 1\right) \underset{c \rightarrow \infty}{\stackrel{d}{\longrightarrow}} \frac{1}{2} B^{a}\left(r_{1}\right)^{2}
\end{aligned}
$$


Next, for the second term,

$$
\begin{aligned}
& \mathbb{E} e^{-2 c \int_{r_{1}}^{r_{2}} f(a) d a}\left[2 c W^{a}\left(r_{1}\right) \int_{r_{1}}^{r_{2}} f(r) e^{c \int_{r_{1}}^{r} f(a) d a} \int_{r_{1}}^{r} e^{c \int_{s}^{r} f(a) d a} d W(s) d r\right] \\
& =2 c \mathbb{E} W^{a}\left(r_{1}\right) \mathbb{E} \int_{r_{1}}^{r_{2}} f(r) e^{-c \int_{r}^{r_{2}} f(a) d a} \int_{r_{1}}^{r} e^{-c\left(\int_{r_{1}}^{s}+\int_{r}^{r_{2}}\right) f(a) d a} d W(s) d r=0,
\end{aligned}
$$

and by Eq 36 ,

$$
\begin{aligned}
& \mathbb{E}\left[e^{-2 c \int_{r_{1}}^{r_{2}} f(a) d a} 2 c W^{a}\left(r_{1}\right) \int_{r_{1}}^{r_{2}} f(r) e^{c \int_{r_{1}}^{r} f(a) d a} \int_{r_{1}}^{r} e^{c \int_{s}^{r} f(a) d a} d W(s) d r\right]^{2} \\
& =r_{1} \int_{r_{1}}^{r_{2}}\left(e^{-2 c \int_{r_{1}}^{s} f(a) d a}+e^{-2 c \int_{r_{1}}^{r_{2}} f(a) d a-2 c \int_{s}^{r_{2}} f(a) d a}-2 e^{-2 c \int_{r_{1}}^{r_{2}} f(a) d a}\right) d r \underset{c \rightarrow \infty}{\longrightarrow} 0 .
\end{aligned}
$$

Finally, by Eq. 38 and 44 .

$$
\begin{aligned}
& \mathbb{E}\left[e^{-2 c \int_{r_{1}}^{r_{2}} f(a) d a} \omega^{2} c \int_{r_{1}}^{r_{2}} f(r)\left(\int_{r_{1}}^{r} e^{c \int_{s}^{r} f(a) d a} d W(s)\right)^{2} d r\right] \\
& =\frac{\omega^{2}}{2} \int_{r_{1}}^{r_{2}} e^{-2 c \int_{r_{1}}^{s} f(a) d a} d s-\frac{\omega^{2}}{2}\left(r_{2}-r_{1}\right) e^{-2 c \int_{r_{1}}^{r_{2}} f(a) d a} \underset{c \rightarrow \infty}{\longrightarrow} 0,
\end{aligned}
$$

and

$$
\begin{aligned}
& \mathbb{V}\left[e^{-2 c \int_{r_{1}}^{r_{2}} f(a) d a} c \int_{r_{1}}^{r_{2}} f(r)\left(\int_{r_{1}}^{r} e^{c \int_{s}^{r} f(a) d a} d W(s)\right)^{2} d r\right] \\
& =\frac{1}{2}\left(\int_{r_{1}}^{r_{2}} e^{-2 c \int_{r_{1}}^{s} f(a) d a} d s\right)^{2}-e^{-2 c \int_{r_{1}}^{r_{2}} f(a) d a} \int_{r_{1}}^{r_{2}} \int_{r_{1}}^{s} e^{-2 c \int_{r_{1}}^{s^{\prime}} f(a) d a} d s^{\prime} d s \\
& -e^{-2 c \int_{r_{1}}^{r_{2}} f(a) d a} \int_{r_{1}}^{r_{2}}\left(r_{2}-s\right) e^{-2 c \int_{r_{1}}^{s} f(a) d a} d s \\
& +e^{-2 c \int_{r_{1}}^{r_{2}} f(a) d a} \int_{r_{1}}^{r_{2}} \int_{r_{1}}^{s} e^{-2 c\left(\int_{r_{1}}^{s^{\prime}}+\int_{s}^{r_{2}}\right) f(a) d a} d s^{\prime} d s \underset{c \rightarrow \infty}{\longrightarrow} 0 .
\end{aligned}
$$

Combining these three terms gives

$$
e^{-2 c \int_{r_{1}}^{r_{2}} f(a) d a} \int_{r_{1}}^{r_{2}} c f(r) F_{c}(r)^{2} d r \underset{c \rightarrow \infty}{\stackrel{d}{\longrightarrow}} \frac{1}{2} B^{a}\left(r_{1}\right)^{2} .
$$

Further, combining Eq. 52, Eq. 53, and Eq. 55, we get

$$
\begin{aligned}
& \frac{e^{-2 c \int_{r_{1}}^{r_{2}} f(a) d a}\left[\int_{0}^{1} F_{c}(r) d B(r)+\lambda+c \int_{r_{1}}^{r_{2}} f(r) F_{c}(r)^{2} d r\right]}{e^{-2 c \int_{r_{1}}^{r_{2}} f(a) d a} \int_{0}^{1} F_{c}(r)^{2} d r} \\
& \underset{c \rightarrow \infty}{\stackrel{d}{\longrightarrow}} \frac{\frac{1}{2} B^{a}\left(r_{1}\right)^{2}}{\left(1-r_{2}\right)\left(B^{a}\left(r_{1}\right)\right)^{2}}=\frac{1}{2\left(1-r_{2}\right)},
\end{aligned}
$$


so that

$$
n(\hat{\theta}-1) \underset{(c, n)_{s e q} \rightarrow \infty}{\stackrel{p}{\longrightarrow}} \frac{1}{2\left(1-r_{2}\right)},
$$

giving a constant in the limit.

The limit behavior of the $t$ statistic for $\hat{\theta}_{O L S}$ follows as in previous results. In particular, we have

$$
\begin{gathered}
n(\hat{\theta}-1) \frac{d}{(c, n)_{s e q} \rightarrow \infty} \frac{1}{2\left(1-r_{2}\right)} \\
e^{-c \int_{r_{1}}^{r_{2}} f(a) d a}\left(\int_{0}^{1} F_{c}(r)^{2} d r\right)^{1 / 2} \underset{c \rightarrow \infty}{\stackrel{d}{\longrightarrow}} \sqrt{1-r_{2}}\left|B^{a}\left(r_{1}\right)\right| .
\end{gathered}
$$

leading to

$$
e^{-c \int_{r_{1}}^{r_{2}} f(a) d a} t_{\hat{\theta}_{O L S}} \underset{(c, n)_{s e q} \rightarrow \infty}{\longrightarrow} \frac{1}{2\left(1-r_{2}\right)} \sqrt{1-r_{2}}\left|B^{a}\left(r_{1}\right)\right| \frac{1}{\sigma}=\frac{\sqrt{r_{1}} \omega}{2 \sigma \sqrt{1-r_{2}}}|\mathcal{N}(0,1)| .
$$

\section{Proof of Theorem 3 ,}

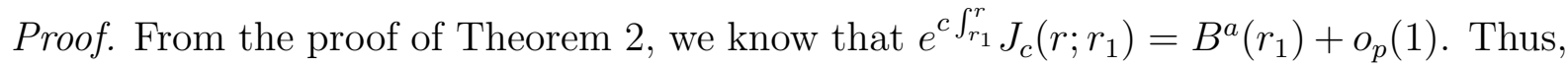
$J_{c}\left(r_{2} ; r_{1}\right)$ has the largest stochastic order among the $J_{c}\left(r ; r_{1}\right)$ for $r \leq r_{2}$. It is convenient to rewrite $J_{c}\left(r ; r_{1}\right)$ in terms of $J_{c}\left(r_{2} ; r_{1}\right)$ as

$$
J_{c}\left(r ; r_{1}\right)=\int_{0}^{r} e^{c \int_{s}^{r} f(a) d a} d B(s)=e^{-c \int_{r}^{r_{2}} f(a) d a} J_{c}\left(r_{2} ; r_{1}\right)-\int_{r}^{r_{2}} e^{-c \int_{r}^{s} f(a) d a} d B(s),
$$

whose first term is exponentially large and whose second term is $O_{p}\left(c^{-0.5}\right)$, since it is Gaussian with zero mean and variance $\int_{r}^{r_{2}} e^{-2 c \int_{r}^{s} f(a) d a} d s=\frac{1}{2 c f(r)}[1+o(1)]$ as $c \rightarrow \infty$, where the asymptotic representation of the integral follows directly from Lemma 2. Next define $\xi_{r}:=\int_{r}^{r_{2}} e^{-c \int_{r}^{s} f(a) d a} d B(s) \sqrt{2 c f(r)} \underset{c \rightarrow \infty}{\stackrel{d}{\longrightarrow}} \mathcal{N}(0,1)$ and rewrite both numerator and denominator of $\left(\int_{0}^{1} F_{c}(r) d B(r)+\lambda+\int_{r_{1}}^{r_{2}} c f(r) F_{c}^{2}(r) d r\right) / \int_{0}^{1} F_{c}^{2}(r) d r$ in terms of $J_{c}\left(r_{2} ; r_{1}\right)$ as follows.

$$
\begin{aligned}
\int_{0}^{1} F_{c}(r) d B(r) & =\int_{0}^{r_{1}} B^{a}(r) d B^{a}(r)+\int_{r_{1}}^{r_{2}} J_{c}\left(r ; r_{1}\right) d B(r)+\int_{r_{2}}^{1}\left(B^{b}(r)+J_{c}\left(r_{2} ; r_{1}\right)\right) d B^{b}(r) \\
& =J_{c}\left(r_{2} ; r_{1}\right)\left(B^{b}(1)-B^{b}\left(r_{2}\right)\right)+J_{c}\left(r_{2} ; r_{1}\right) \int_{r_{1}}^{r_{2}} e^{-c \int_{r}^{r_{2}} f(a) d a} d B(s)+O_{p}(1),
\end{aligned}
$$




$$
\begin{aligned}
\int_{r_{1}}^{r_{2}} c f(r) F_{c}^{2}(r) d r & =\int_{r_{1}}^{r_{2}} c f(r) J_{c}^{2}\left(r ; r_{1}\right) d r=J_{c}^{2}\left(r_{2} ; r_{1}\right) c \int_{r_{1}}^{r_{2}} f(r) e^{-2 c \int_{r}^{r_{2}} f(a) d a} d r \\
& -\frac{2 c J_{c}\left(r_{2} ; r_{1}\right)}{\sqrt{2 c}} \int_{r_{1}}^{r_{2}} \xi_{r} \sqrt{f(r)} e^{-c \int_{r}^{r_{2}} f(a) d a} d r+\frac{1}{2} \int_{r_{1}}^{r_{2}} \xi_{r}^{2} d r
\end{aligned}
$$

where

$$
\begin{array}{r}
\mathbb{E}\left(\int_{r_{1}}^{r_{2}} \xi_{r} \sqrt{f(r)} e^{-c \int_{r}^{r_{2}} f(a) d a} d r\right)^{2}=\mathbb{E} \int_{r_{1}}^{r_{2}} \int_{r_{1}}^{r_{2}} \xi_{r} \xi_{t} \sqrt{f(r) f(t)} e^{-c \int_{r}^{r_{2}} f(a) d a} e^{-c \int_{t}^{r_{2}} f(a) d a} d t d r \\
\unlhd 1 \int_{r_{1}}^{r_{2}} \int_{r_{1}}^{r_{2}} \sqrt{f(r) f(t)} e^{-c \int_{r}^{r_{2}} f(a) d a} e^{-c \int_{t}^{r_{2}} f(a) d a} d t d r=\left(\int_{r_{1}}^{r_{2}} \sqrt{f(r)} e^{-c \int_{r}^{r_{2}} f(a) d a} d r\right)^{2} \\
=\frac{1}{(2 c)^{2} f\left(r_{2}\right)}[1+o(1)],
\end{array}
$$

again by Lemma 2. Hence, $\int_{r_{1}}^{r_{2}} \xi_{r} \sqrt{f(r)} e^{-c \int_{r}^{r_{2}} f(a) d a} d r=O_{p}(1 / c)$. Noting that $c \int_{r_{1}}^{r_{2}} f(r) e^{-2 c \int_{r}^{r_{2}} f(a) d a} d r=\frac{1}{2}\left(1-e^{-2 c \int_{r_{1}}^{r_{2}} f(a) d a}\right)$, we then have

$$
\int_{r_{1}}^{r_{2}} c f(r) F_{c}^{2}(r) d r=\frac{1}{2}\left(1-e^{-2 c \int_{r_{1}}^{r_{2}} f(a) d a}\right) J_{c}^{2}\left(r_{2} ; r_{1}\right)+J_{c}\left(r_{2} ; r_{1}\right) O_{p}(1 / \sqrt{c})
$$

and

$$
\begin{aligned}
\int_{0}^{1} F_{c}^{2}(r) d r & =\int_{0}^{r_{1}} B^{a}(r)^{2} d r+\int_{r_{1}}^{r_{2}} J_{c}^{2}\left(r ; r_{1}\right) d r+\int_{r_{2}}^{1}\left(B^{b}(r)+J_{c}\left(r_{2} ; r_{1}\right)\right)^{2} d r \\
& =J_{c}^{2}\left(r_{2} ; r_{1}\right) \int_{r_{1}}^{r_{2}} e^{-2 c \int_{r}^{r_{2}} f(a) d a} d r-\frac{2 J_{c}\left(r_{2} ; r_{1}\right)}{\sqrt{2 c}} \int_{r_{1}}^{r_{2}} \frac{\xi_{r}}{\sqrt{f(r)}} e^{-c \int_{r}^{r_{2}} f(a) d a} d r \\
& +\frac{1}{2 c} \int_{r_{1}}^{r_{2}} \frac{\xi_{r}^{2}}{f(r)} d r+\left(1-r_{2}\right) J_{c}^{2}\left(r_{2} ; r_{1}\right)+2 J_{c}\left(r_{2} ; r_{1}\right) \int_{r_{2}}^{1} B^{b}(r) d r+O_{p}(1) \\
& =J_{c}^{2}\left(r_{2} ; r_{1}\right)\left(1-r_{2}+\int_{r_{1}}^{r_{2}} e^{-2 c \int_{r}^{r_{2}} f(a) d a} d r\right)+2 J_{c}\left(r_{2} ; r_{1}\right) \int_{r_{2}}^{1} B^{b}(r) d r \\
& +J_{c}\left(r_{2} ; r_{1}\right) O_{p}(1 / c \sqrt{c})+O_{p}(1),
\end{aligned}
$$

where $\frac{1}{2 c} \int_{r_{1}}^{r_{2}} \frac{\xi_{r}^{2}}{f(r)} d r \leq \frac{1}{2 C c} \int_{r_{1}}^{r_{2}} \xi_{r}^{2} d r=O_{p}(1 / c)$. Combining Eq. (57), (58), and (59), we have as $c \rightarrow \infty$

$$
{ }^{1} \mathbb{E} \xi_{r} \xi_{t} \leq \sqrt{\mathbb{E} \xi_{r}^{2} \mathbb{E} \xi_{t}^{2}}=\sqrt{1 \cdot 1}=1
$$




$$
\begin{aligned}
& \frac{\int_{0}^{1} F_{c}(r) d B(r)+\lambda+\int_{r_{1}}^{r_{2}} c f(r) F_{c}^{2}(r) d r}{\int_{0}^{1} F_{c}^{2}(r) d r} \\
& =\frac{\frac{1}{2}\left(1-e^{-2 c \int_{r_{1}}^{r_{2}} f(a) d a}\right) J_{c}^{2}\left(r_{2} ; r_{1}\right)+J_{c}\left(r_{2} ; r_{1}\right)\left(B^{b}(1)-B^{b}\left(r_{2}\right)\right)}{J_{c}^{2}\left(r_{2} ; r_{1}\right)\left(1-r_{2}+\int_{r_{1}}^{r_{2}} e^{-2 c \int_{r}^{r_{2}} f(a) d a} d r\right)+2 J_{c}\left(r_{2} ; r_{1}\right) \int_{r_{2}}^{1} B^{b}(r) d r}\left[1+o_{p}(1)\right] \\
& =\frac{\frac{1}{2}\left(1-e^{-2 c \int_{r_{1}}^{r_{2}} f(a) d a}\right)}{1-r_{2}+\int_{r_{1}}^{r_{2}} e^{-2 c \int_{r}^{r_{2}} f(a) d a} d r} \times \frac{1+\frac{\left(B^{b}(1)-B^{b}\left(r_{2}\right)\right)}{\frac{1}{2}\left(1-e^{-2 c \int_{r_{1}}^{r_{2}} f(a) d a}\right) J_{c}\left(r_{2} ; r_{1}\right)}}{\left(1-r_{2}+\int_{r_{1}}^{r_{2}} e^{\left.-2 c \int_{r}^{r_{2} f(a) d a} d r\right) J_{c}\left(r_{2} ; r_{1}\right)}\right.} \\
& =\frac{\frac{1}{2}\left(1-e^{-2 c \int_{r_{1}}^{r_{2}} f(a) d a}\right)}{1-r_{2}+\int_{r_{1}}^{r_{2}} e^{-2 c \int_{r}^{r_{2}} f(a) d a} d r} \times\left(1+\frac{\left(B^{b}(1)-B^{b}\left(r_{2}\right)\right)}{\frac{1}{2}\left(1-e^{-2 c \int_{r_{1}}^{r_{2}} f(a) d a}\right) J_{c}\left(r_{2} ; r_{1}\right)}\right. \\
& -\frac{2 \int_{r_{2}}^{1} B^{b}(r) d r}{\left(1-r_{2}+\int_{r_{1}}^{r_{2}} e^{-2 c \int_{r}^{r_{2}} f(a) d a} d r\right)} J_{c}\left(r_{2} ; r_{1}\right)
\end{aligned}
$$

Rewriting Eq. (60) and using the fact that $J_{c}\left(r_{2} ; r_{1}\right)=e^{c \int_{r_{1}}^{r_{2}} f(a) d a} B^{a}\left(r_{1}\right)+O_{p}(1)$, we get

$$
\begin{aligned}
& e^{c \int_{r_{1}}^{r_{2}} f(a) d a}\left(\frac{\int_{0}^{1} F_{c}(r) d B(r)+\lambda+\int_{r_{1}}^{r_{2}} c f(r) F_{c}^{2}(r) d r}{\int_{0}^{1} F_{c}^{2}(r) d r}-\frac{\frac{1}{2}\left(1-e^{-2 c \int_{r_{1}}^{r_{2}} f(a) d a}\right)}{1-r_{2}+\int_{r_{1}}^{r_{2}} e^{-2 c \int_{r}^{r_{2}} f(a) d a} d r}\right) \\
& =\frac{1}{B^{a}\left(r_{1}\right)}\left(\frac{B^{b}(1)-B^{b}\left(r_{2}\right)}{\frac{1}{2}\left(1-e^{-2 c \int_{r_{1}}^{r_{2}} f(a) d a}\right)}-\frac{2 \int_{r_{2}}^{1} B^{b}(r) d r}{1-r_{2}+\int_{r_{1}}^{r_{2}} e^{-2 c \int_{r}^{r_{2}} f(a) d a} d r}\right) \\
& \times \frac{\frac{1}{2}\left(1-e^{-2 c \int_{r_{1}}^{r_{2}} f(a) d a}\right)}{1-r_{2}+\int_{r_{1}}^{r_{2}} e^{-2 c \int_{r}^{r_{2}} f(a) d a} d r}\left[1+o_{p}(1)\right] \\
& \quad \underset{c \rightarrow \infty}{\stackrel{d}{\longrightarrow} \frac{1}{2\left(1-r_{2}\right) B^{a}\left(r_{1}\right)}}\left(2\left(B^{b}(1)-B^{b}\left(r_{2}\right)\right)-\frac{2 \int_{r_{2}}^{1} B^{b}(r) d r}{1-r_{2}}\right) .
\end{aligned}
$$


Because $2\left(B^{b}(1)-B^{b}\left(r_{2}\right)\right)-\frac{2 \int_{r_{2}}^{1} B^{b}(r) d r}{1-r_{2}}$ is Gaussian and independent of $B^{a}\left(r_{1}\right)$ the limit variate in (62) has a Cauchy distribution. We proceed to calculate the scale coefficient of this distribution. The component terms in the variance of the numerator are as follows:

$$
\begin{aligned}
\mathbb{E}\left(2\left(B^{b}(1)-B^{b}\left(r_{2}\right)\right) \frac{2}{1-r_{2}} \int_{r_{2}}^{1} B^{b}(r) d r\right) & =\frac{4}{1-r_{2}} \mathbb{E} B\left(1-r_{2}\right) \int_{0}^{1-r_{2}} B(r) d r \\
& =\frac{4 \omega^{2}}{1-r_{2}} \int_{0}^{1-r_{2}} r d r=2\left(1-r_{2}\right) \omega^{2} \\
\mathbb{E}\left(2\left(B^{b}(1)-B^{b}\left(r_{2}\right)\right)\right)^{2} & =4\left(1-r_{2}\right) \omega^{2}, \\
\mathbb{E}\left(\frac{2}{1-r_{2}} \int_{r_{2}}^{1} B^{b}(r) d r\right)^{2} & =\frac{4}{\left(1-r_{2}\right)^{2}} \int_{0}^{1-r_{2}} \int_{0}^{1-r_{2}} \mathbb{E}(B(s) B(r)) d s d r \\
& =\frac{4 \omega^{2}}{\left(1-r_{2}\right)^{2}} \int_{0}^{1-r_{2}} \int_{0}^{1-r_{2}} \min (s, r) d s d r \\
& =\frac{4 \omega^{2}}{\left(1-r_{2}\right)^{2}} \frac{\left(1-r_{2}\right)^{3}}{3}=\frac{4}{3}\left(1-r_{2}\right) \omega^{2}
\end{aligned}
$$

so that $2\left(B^{b}(1)-B^{b}\left(r_{2}\right)\right)-\frac{2 \int_{r_{2}}^{1} B^{b}(r) d r}{1-r_{2}} \sim \mathcal{N}\left(0, \frac{4}{3}\left(1-r_{2}\right) \omega^{2}\right)$ and thus

$$
\begin{aligned}
& e^{c \int_{r_{1}}^{r_{2}} f(a) d a}\left(\frac{\int_{0}^{1} F_{c}(r) d B(r)+\lambda+\int_{r_{1}}^{r_{2}} c f(r) F_{c}^{2}(r) d r}{\int_{0}^{1} F_{c}^{2}(r) d r}-\frac{1-e^{-2 c \int_{r_{1}}^{r_{2}} f(a) d a}}{2\left(1-r_{2}+\int_{r_{1}}^{r_{2}} e^{-2 c \int_{r}^{r_{2}} f(a) d a} d r\right)}\right) \\
& \underset{c \rightarrow+\infty}{\stackrel{d}{\longrightarrow}} \frac{1}{2\left(1-r_{2}\right)} \frac{\sqrt{4 / 3\left(1-r_{2}\right)}}{\sqrt{r_{1}}} \mathcal{C}=\frac{1}{\sqrt{3 r_{1}\left(1-r_{2}\right)}} \mathcal{C} .
\end{aligned}
$$

It follows that

$$
e^{c \int_{r_{1}}^{r_{2}} f(a) d a} n\left(\hat{\theta}_{O L S}-1-\frac{1-e^{-2 c \int_{r_{1}}^{r_{2}} f(a) d a}}{2 n\left(1-r_{2}+\int_{r_{1}}^{r_{2}} e^{-2 c \int_{r}^{r_{2}} f(a) d a} d r\right)}\right) \frac{d}{(n, c)_{s e q} \rightarrow+\infty} \frac{1}{\sqrt{3 r_{1}\left(1-r_{2}\right)}} \mathcal{C} .
$$

\section{Proof of Theorem 4 .}

Proof. The proof of (19) follows by the martingale CLT for triangular arrays by establishing the stability and Lindeberg conditions. First consider the variance of $\frac{1}{\sqrt{k_{n}}} \tilde{X}_{\lfloor n r\rfloor}$, for $r \in(0,1]$, which we denote in martingale conditional variance notation as $\left\langle\frac{1}{\sqrt{k_{n}}} \tilde{X}_{\lfloor n r\rfloor}\right\rangle$. 
Using the martingale difference property of $\left\{u_{t}\right\}_{t}$, we have

$$
\begin{aligned}
\left\langle\frac{1}{\sqrt{k_{n}}} \tilde{X}_{\lfloor n r\rfloor}\right\rangle & =\frac{\sigma^{2}}{k_{n}} \sum_{j=1}^{\lfloor n r\rfloor} e^{-\left\{\frac{2}{k_{n}} \sum_{l=\lfloor n r\rfloor-j+1}^{\lfloor n r\rfloor} c\left(\frac{\lfloor n r\rfloor-l+1}{n}\right)\right\}}+o(1) \\
& =\sigma^{2} \int_{0}^{\frac{\lfloor n r\rfloor}{k_{n}}} e^{\left\{-2 \int_{0}^{z} c\left(x \frac{k_{n}}{n}\right) d x\right\} d z}+o(1) \\
& \underset{n \rightarrow \infty}{\longrightarrow} \sigma^{2} \int_{0}^{\infty} e^{-2 z c(0)} d z=\frac{\sigma^{2}}{2 c(0)},
\end{aligned}
$$

which is constant. Next suppose that $s>r$ and consider the covariance between $\frac{1}{\sqrt{k_{n}}} \tilde{X}_{\lfloor n r\rfloor}$ and $\frac{1}{\sqrt{k_{n}}} \tilde{X}_{\lfloor n s\rfloor}$, denoted by $\left\langle\frac{1}{\sqrt{k_{n}}} \tilde{X}_{\lfloor n r\rfloor}, \frac{1}{\sqrt{k_{n}}} \tilde{X}_{\lfloor n s\rfloor}\right\rangle$. Observe that

$$
\begin{aligned}
& \left\langle\frac{1}{\sqrt{k_{n}}} \tilde{X}_{\lfloor n r\rfloor}, \frac{1}{\sqrt{k_{n}}} \tilde{X}_{\lfloor n s\rfloor}\right\rangle \\
& =\frac{\sigma^{2}}{k_{n}} \sum_{j=1}^{\lfloor n r\rfloor} e^{-\frac{1}{k_{n}}\left(\sum_{l=\lfloor n r\rfloor-j+1}^{\lfloor n r\rfloor} c\left(\frac{\lfloor n r\rfloor-l+1}{n}\right)+\sum_{l=\lfloor n s\rfloor-j+1}^{\lfloor n s\rfloor} c\left(\frac{\lfloor n s\rfloor-l+1}{n}\right)\right)}+o_{p}(1) \\
& =\sigma^{2} \int_{0}^{\frac{\lfloor n r\rfloor}{k_{n}}} e^{-2 \int_{0}^{z} c\left(x \frac{k_{n}}{n}\right) d x} d z+o(1) \\
& \underset{n \rightarrow \infty}{\longrightarrow} \sigma^{2} \int_{0}^{\infty} e^{-2 z c(0)} d z=\frac{\sigma^{2}}{2 c(0)}=\left\langle\frac{1}{\sqrt{k_{n}}} \tilde{X}_{\lfloor n r\rfloor}\right\rangle=\left\langle\frac{1}{\sqrt{k_{n}}} \tilde{X}_{\lfloor n s\rfloor}\right\rangle,
\end{aligned}
$$

is also constant. The Lindeberg condition is established as follows. Take $\delta>0$, and noting that $c(a) \geq 0$ over $a \in[0,1]$ and $c(a) \geq C>0$ for all $a \in[0, \varepsilon] \cup[1-\varepsilon, 1]$ for some $\varepsilon>0$, we obtain

$$
\begin{aligned}
& \sum_{j=1}^{\lfloor n r\rfloor} \mathbb{E}\left\{\frac{e^{-\frac{2}{k_{n}} \sum_{l=\lfloor n r\rfloor-j+1}^{\lfloor n r\rfloor} c\left(\frac{\lfloor n r\rfloor-l+1}{n}\right)} u_{j}^{2}}{k_{n}} \mathbf{1}\left[\left|u_{j}\right|>\delta \sqrt{k_{n}} e^{\frac{1}{k_{n}} \sum_{l=\lfloor n r\rfloor-j+1}^{\lfloor n r\rfloor} c\left(\frac{\lfloor n r\rfloor-l+1}{n}\right)}\right]\right\} \\
= & \frac{1}{k_{n}} \sum_{j=1}^{\lfloor n r\rfloor} e^{-\frac{2}{k_{n}} \sum_{l=\lfloor n r\rfloor-j+1}^{\lfloor n r\rfloor} c\left(\frac{\lfloor n r\rfloor-l+1}{n}\right)} \mathbb{E}\left\{u_{j}^{2} \mathbf{1}\left[\left|u_{j}\right|>\delta \sqrt{k_{n}} e^{\frac{1}{k_{n}} \sum_{l=\lfloor n r\rfloor-j+1}^{\lfloor n r\rfloor} c\left(\frac{\lfloor n r\rfloor-l+1}{n}\right)}\right]\right\} \\
\leq & \frac{1}{k_{n}} \sum_{j=1}^{\lfloor n r\rfloor} e^{-\frac{2}{k_{n}} \sum_{l=\lfloor n r\rfloor-j+1}^{\lfloor n r\rfloor} c\left(\frac{\lfloor n r\rfloor-l+1}{n}\right)} \times \mathbb{E}\left\{u_{1}^{2} \mathbf{1}\left[\left|u_{1}\right|>\delta \sqrt{k_{n}}\right]\right\} \\
= & \frac{1}{n} \frac{n}{k_{n}} \sum_{j=1}^{\lfloor n r\rfloor} e^{-\frac{2 n}{k_{n}} \sum_{l=\lfloor n r\rfloor-j+1}^{\lfloor n r\rfloor} c\left(\frac{\lfloor n r\rfloor-l+1}{n}\right) \frac{1}{n}} \times \mathbb{E}\left\{u_{1}^{2} \mathbf{1}\left[\left|u_{1}\right|>\delta \sqrt{k_{n}}\right]\right\}
\end{aligned}
$$


BOUNDARY LIMIT THEORY FOR FUNCTIONAL

$$
\begin{aligned}
& =\frac{n}{k_{n}} \int_{0}^{r} e^{-\frac{2 n}{k_{n}} \int_{0}^{s} c(a) d a} d s \times \mathbb{E}\left\{u_{1}^{2} \mathbf{1}\left[\left|u_{1}\right|>\delta \sqrt{k_{n}}\right]\right\}[1+o(1)] \\
& =\frac{1}{2 c(0)} \mathbb{E}\left\{u_{1}^{2} \mathbf{1}\left[\left|u_{1}\right|>\delta \sqrt{k_{n}}\right]\right\}[1+o(1)] \rightarrow 0
\end{aligned}
$$

since $\mathbb{E}\left(u_{1}^{2}\right)<\infty$ and by Lemma 2 ,

$$
\int_{0}^{r} e^{-\frac{2 n}{k_{n}} \int_{0}^{s} c(a) d a} d s=\frac{k_{n}}{2 n c(0)}[1+o(1)] .
$$

Thus, the Lindeberg condition holds and (19) holds for all $r \in(0,1]$. Result 20 follows in a related way and the proof is omitted.

\section{Proof of Theorem 5 .}

Proof. The proof is similar to the line of reasoning used in the proof of Theorem 3 but the calculations are considerably more complex. First, it is useful to re-normalize the time series $X_{t}$ as $\tilde{X}_{t}$, and then rewrite everything in terms of the last observation, $\tilde{X}_{n}$. Note that

$$
\begin{aligned}
\tilde{X}_{t} & =e^{-\frac{1}{k_{n}} \sum_{l=1}^{t} c\left(\frac{t-l+1}{n}\right)} X_{t}=e^{-\frac{1}{k_{n}} \sum_{j=1}^{t} c\left(\frac{j}{n}\right)} X_{t}=X_{0}+\sum_{j=1}^{t} e^{-\frac{1}{k_{n}} \sum_{l=1}^{j} c(l / n)} u_{j} \\
& =\tilde{X}_{n}-\sum_{j=t+1}^{n} e^{-\frac{1}{k_{n}} \sum_{l=1}^{j} c(l / n)} u_{j} .
\end{aligned}
$$

We start with the decomposition

$$
k_{n}\left(\hat{\theta}_{O L S}-1\right)=\frac{\frac{1}{n} \sum_{t=1}^{n} X_{t-1} \Delta X_{t}}{\frac{1}{n k_{n}} \sum_{t=1}^{n} X_{t-1}^{2}}=\frac{\frac{1}{n} \sum_{t=1}^{n} \frac{c\left(\frac{t}{n}\right)}{k_{n}} X_{t-1}^{2}}{\frac{1}{n k_{n}} \sum_{t=1}^{n} X_{t-1}^{2}}+\frac{\frac{1}{n} \sum_{t=1}^{n} X_{t-1} u_{t}}{\frac{1}{n k_{n}} \sum_{t=1}^{n} X_{t-1}^{2}},
$$

and rewrite the component sums in terms of $\tilde{X}_{n}$ using 64. We consider each term separately, starting with the common denominator.

(i) Denominator of (65)

Upon scaling by $e^{-\frac{2}{k_{n}} \sum_{j=1}^{n} c\left(\frac{s}{n}\right)}$, we write the denominator as

$$
\begin{aligned}
& e^{-\frac{2}{k_{n}} \sum_{j=1}^{n} c\left(\frac{j}{n}\right)} \frac{1}{n} \sum_{t=1}^{n}\left(\frac{X_{t-1}}{\sqrt{k_{n}}}\right)^{2}=\frac{1}{n} \sum_{t=1}^{n}\left(\frac{\tilde{X}_{t-1}}{\sqrt{k_{n}}}\right)^{2} \frac{e^{\frac{2}{k_{n}} \sum_{j=1}^{t-1} c\left(\frac{j}{n}\right)}}{e^{\frac{2}{k_{n}} \sum_{j=1}^{n} c\left(\frac{j}{n}\right)}} \\
& =\frac{1}{n} \sum_{t=1}^{n}\left(\frac{\tilde{X}_{n}-\sum_{j=t}^{n} e^{-\frac{1}{k_{n}} \sum_{l=1}^{j} c(l / n)} u_{j}}{\sqrt{k_{n}}}\right)^{-\frac{2}{k_{n}} \sum_{j=t}^{n} c\left(\frac{j}{n}\right)}
\end{aligned}
$$




$$
\begin{aligned}
& =\frac{1}{n}\left(\frac{\tilde{X}_{n}}{\sqrt{k_{n}}}\right)^{2} \sum_{t=1}^{n} e^{-\frac{2}{k_{n}} \sum_{j=t}^{n} c\left(\frac{j}{n}\right)}-\frac{2}{n} \frac{\tilde{X}_{n}}{\sqrt{k_{n}}} \sum_{t=1}^{n} e^{-\frac{2}{k_{n}} \sum_{j=t}^{n} c\left(\frac{j}{n}\right)} \sum_{j=t}^{n} e^{-\frac{1}{k_{n}} \sum_{l=1}^{j} c(l / n)} \frac{u_{j}}{\sqrt{k_{n}}} \\
& +\frac{1}{n} \sum_{t=1}^{n} e^{-\frac{2}{k_{n}} \sum_{j=t}^{n} c\left(\frac{j}{n}\right)}\left(\sum_{j=t}^{n} e^{-\frac{1}{k_{n}} \sum_{l=1}^{j} c(l / n)} \frac{u_{j}}{\sqrt{k_{n}}}\right)^{2}=: D_{n, 1}+D_{n, 2}+D_{n, 3} .
\end{aligned}
$$

From Theorem 4, we know that $\frac{\tilde{X}_{n}}{\sqrt{k_{n}}}=O_{p}(1)$. It is easy to see that in the last part of (66) the first term $D_{n, 1}$ has the largest order of magnitude and the second term $D_{n, 2}$ has the second largest order. More specifically, the three terms of 66 take the following forms as $\frac{1}{k_{n}}+\frac{k_{n}}{n} \rightarrow 0$ :

$$
\begin{aligned}
& D_{n, 1}=\frac{1}{n}\left(\frac{\tilde{X}_{n}}{\sqrt{k_{n}}}\right)^{2} \sum_{t=1}^{n} e^{-\frac{2}{k_{n}} \sum_{j=t}^{n} c\left(\frac{j}{n}\right)}=\left(\frac{\tilde{X}_{n}}{\sqrt{k_{n}}}\right)^{2} \int_{0}^{1} e^{-\frac{2 n}{k_{n}} \int_{s}^{1} c(a) d a} d s\left[1+o_{p}(1)\right] \\
& =\left(\frac{\tilde{X}_{n}}{\sqrt{k_{n}}}\right)^{2} \frac{k_{n}}{2 n c(1)}\left[1+o_{p}(1)\right]
\end{aligned}
$$

by Lemma 2; and similarly

$$
\begin{aligned}
& D_{n, 2}=\frac{2}{n} \frac{\tilde{X}_{n}}{\sqrt{k_{n}}} \sum_{t=1}^{n} e^{-\frac{2}{k_{n}} \sum_{j=t}^{n} c\left(\frac{j}{n}\right)} \sum_{j=t}^{n} e^{-\frac{1}{k_{n}} \sum_{l=1}^{j} c(l / n)} \frac{u_{j}}{\sqrt{k_{n}}} \\
&=\frac{2 \tilde{X}_{n}}{\sqrt{k_{n}}} \sqrt{\frac{n}{k_{n}}} \int_{0}^{1} e^{-\frac{2 n}{k_{n}} \int_{z}^{1} c(a) d a} \int_{z}^{1} e^{-\frac{n}{k_{n}} \int_{0}^{s} c(a) d a} d B(s) d z\left[1+o_{p}(1)\right] \\
&=\frac{2 \tilde{X}_{n}}{\sqrt{k_{n}}} \sqrt{\frac{n}{k_{n}}} e^{-\frac{n}{k_{n}} \int_{0}^{1} c(a) d a} \int_{0}^{1} e^{-\frac{n}{k_{n}} \int_{z}^{1} c(a) d a} \int_{z}^{1} e^{-\frac{n}{k_{n}} \int_{z}^{s} c(a) d a} d B(s) d z \\
&=\frac{2 \tilde{X}_{n}}{\sqrt{k_{n}}} \sqrt{\frac{n}{k_{n}}} e^{-\frac{n}{k_{n}} \int_{0}^{1} c(a) d a} \int_{0}^{1} e^{-\frac{n}{k_{n}} \int_{s}^{1} c(a) d a} \int_{0}^{s} e^{-\frac{2 n}{k_{n}} \int_{z}^{s} c(a) d a} d z d B(s) \\
&=\frac{2 \tilde{X}_{n}}{\sqrt{k_{n}}} \sqrt{\frac{k_{n}}{n}} e^{-\frac{n}{k_{n}} \int_{0}^{1} c(a) d a} \int_{0}^{1} e^{-\frac{n}{k_{n}} \int_{s}^{1} c(a) d a} \frac{1}{2 c(s)} d B(s)\left[1+o_{p}(1)\right] \\
&= \frac{2 \tilde{X}_{n}}{\sqrt{k_{n}}} \frac{k_{n}}{n} e^{-\frac{n}{k_{n}} \int_{0}^{1} c(a) d a} \sqrt{\frac{n}{k_{n}}} \int_{0}^{1} e^{-\frac{n}{k_{n}} \int_{s}^{1} c(a) d a} \frac{1}{2 c(s)} d B(s), \\
&= O_{p}\left(\frac{k_{n}}{n} e^{-\frac{n}{k_{n}} \int_{0}^{1} c(a) d a} ;\right. \\
&
\end{aligned}
$$


and finally

$$
\begin{aligned}
& D_{n, 3}=\frac{1}{n} \sum_{t=1}^{n} e^{-\frac{2}{k_{n}} \sum_{j=t}^{n} c\left(\frac{j}{n}\right)}\left(\sum_{j=t}^{n} e^{-\frac{1}{k_{n}} \sum_{l=1}^{j} c(l / n)} \frac{u_{j}}{\sqrt{k_{n}}}\right)^{2} \\
& \frac{n}{k_{n}} \int_{0}^{1} e^{-\frac{2 n}{k_{n}} \int_{z}^{1} c(a) d a}\left(\int_{z}^{1} e^{-\frac{n}{k_{n}} \int_{0}^{s} c(a) d a} d B(s)\right)^{2} d z\left[1+o_{p}(1)\right] \\
& =\frac{n}{k_{n}} e^{-\frac{2 n}{k_{n}} \int_{0}^{1} c(a) d a} \int_{0}^{1}\left(\int_{z}^{1} e^{-\frac{n}{k_{n}} \int_{z}^{s} c(a) d a} d B(s)\right)^{2} d z \\
& =e^{-\frac{2 n}{k_{n}} \int_{0}^{1} c(a) d a} \int_{0}^{1}\left(\sqrt{\frac{n}{k_{n}}} \int_{z}^{1} e^{-\frac{n}{k_{n}} \int_{z}^{s} c(a) d a} d B(s)\right)^{2} d z \\
& =O_{p}\left(e^{-\frac{2 n}{k_{n}} \int_{0}^{1} c(a) d a}\right) .
\end{aligned}
$$

Note that the stochastic integral representation $\int_{0}^{1} e^{-\frac{n}{k_{n}} \int_{s}^{1} c(a) d a} \frac{1}{2 c(s)} d B(s)$ that appears in the Eq. (68) relies as $n \rightarrow \infty$ on the function $c(s)$ only in the immediate neighborhood of the end point $s=1$, so asymptotically the condition $c(1)>0$ is sufficient for the asymptotics to hold.

\section{(ii) Numerators of (65)}

Start by considering the numerator in the first term on the right side of (65). Upon scaling by $e^{-\frac{2}{k_{n}} \sum_{j=1}^{n} c\left(\frac{j}{n}\right)}$, as for the denominator, and by expanding the expression in terms of $\tilde{X}_{n}$, we obtain

$$
\begin{aligned}
& e^{-\frac{2}{k_{n}} \sum_{j=1}^{n} c\left(\frac{j}{n}\right)} \frac{1}{n} \sum_{t=1}^{n} c\left(\frac{t}{n}\right)\left(\frac{X_{t-1}}{\sqrt{k_{n}}}\right)^{2}=\frac{1}{n} \sum_{t=1}^{n} c\left(\frac{t}{n}\right)\left(\frac{\tilde{X}_{t-1}}{\sqrt{k_{n}}}\right)^{2} e^{-\frac{2}{k_{n}} \sum_{j=t}^{n} c\left(\frac{j}{n}\right)} \\
& =\frac{1}{n}\left(\frac{\tilde{X}_{n}}{\sqrt{k_{n}}}\right)^{2} \sum_{t=1}^{n} c\left(\frac{t}{n}\right) e^{-\frac{2}{k_{n}} \sum_{j=t}^{n} c\left(\frac{j}{n}\right)}-\frac{2}{n} \frac{\tilde{X}_{n}}{\sqrt{k_{n}}} \sum_{t=1}^{n} c\left(\frac{t}{n}\right) e^{-\frac{2}{k_{n}} \sum_{j=t}^{n} c\left(\frac{j}{n}\right)} \sum_{j=t}^{n} e^{-\frac{1}{k_{n}} \sum_{l=1}^{j} c\left(\frac{l}{n}\right)} \frac{u_{j}}{\sqrt{k_{n}}} \\
& +\frac{1}{n} \sum_{t=1}^{n} c\left(\frac{t}{n}\right) e^{-\frac{2}{k_{n}} \sum_{j=t}^{n} c\left(\frac{j}{n}\right)}\left(\sum_{j=t}^{n} e^{-\frac{1}{k_{n}} \sum_{l=1}^{j} c(l / n)} \frac{u_{j}}{\sqrt{k_{n}}}\right)^{2} \\
& =: N_{n, 1}^{A}+N_{n, 2}^{A}+N_{n, 3}^{A} .
\end{aligned}
$$

By the same logic employed with the denominator, in 70 the first term has the largest order of magnitude, and the second has the second largest order of magnitude. Note, in 
particular, that

$$
\begin{aligned}
& N_{n, 2}^{A}=\frac{2}{n} \frac{\tilde{X}_{n}}{\sqrt{k_{n}}} \sum_{t=1}^{n} c\left(\frac{t}{n}\right) e^{-\frac{2}{k_{n}} \sum_{j=t}^{n} c\left(\frac{j}{n}\right)} \sum_{j=t}^{n} e^{-\frac{1}{k_{n}} \sum_{l=1}^{j} c\left(\frac{l}{n}\right)} \frac{u_{j}}{\sqrt{k_{n}}} \\
&=\frac{2 \tilde{X}_{n}}{\sqrt{k_{n}}} \sqrt{\frac{n}{k_{n}}} \int_{0}^{1} c(z) e^{-\frac{2 n}{k_{n}} \int_{z}^{1} c(a) d a} \int_{z}^{1} e^{-\frac{n}{k_{n}} \int_{0}^{s} c(a) d a} d B(s) d z\left[1+o_{p}(1)\right] \\
&=\frac{2 \tilde{X}_{n}}{\sqrt{k_{n}}} \sqrt{\frac{n}{k_{n}}} e^{-\frac{n}{k_{n}} \int_{0}^{1} c(a) d a} \int_{0}^{1} c(z) e^{-\frac{n}{k_{n}} \int_{z}^{1} c(a) d a} \int_{z}^{1} e^{-\frac{n}{k_{n}} \int_{z}^{s} c(a) d a} d B(s) d z \\
&=\frac{2 \tilde{X}_{n}}{\sqrt{k_{n}}} \sqrt{\frac{n}{k_{n}}} e^{-\frac{n}{k_{n}} \int_{0}^{1} c(a) d a} \int_{0}^{1} e^{-\frac{n}{k_{n}} \int_{s}^{1} c(a) d a} \int_{0}^{s} c(z) e^{-\frac{2 n}{k_{n}} \int_{z}^{s} c(a) d a} d z d B(s), \\
&=\frac{2 \tilde{X}_{n}}{\sqrt{k_{n}}} \sqrt{\frac{k_{n}}{n}} e^{-\frac{n}{k_{n}} \int_{0}^{1} c(a) d a} \int_{0}^{1} e^{-\frac{n}{k_{n}} \int_{s}^{1} c(a) d a} \frac{1}{2} d B(s)\left[1+o_{p}(1)\right] \\
&=\frac{\tilde{X}_{n}}{\sqrt{k_{n}}} \frac{k_{n}}{n} e^{-\frac{n}{k_{n}} \int_{0}^{1} c(a) d a} \sqrt{\frac{n}{k_{n}}} \int_{0}^{1} e^{-\frac{n}{k_{n}} \int_{s}^{1} c(a) d a} d B(s), \\
&= O_{p}\left(\frac{k_{n}}{n} e^{-\frac{n}{k_{n}} \int_{0}^{1} c(a) d a}\right)
\end{aligned}
$$

which has precisely the same form and consequently the same order as (68). Similar arguments to those of the denominator apply to the other two terms in this numerator expansion.

Next consider the numerator of the second component of $(65)$. Scaling this term by $\frac{n}{k_{n}} e^{-\frac{1}{k_{n}} \sum_{j=1}^{n} c\left(\frac{j}{n}\right)}$, we have

$$
\begin{aligned}
& e^{-\frac{1}{k_{n}} \sum_{j=1}^{n} c\left(\frac{j}{n}\right)} \sum_{t=1}^{n} \frac{X_{t-1}}{\sqrt{k_{n}}} \frac{u_{t}}{\sqrt{k_{n}}}=\sum_{t=1}^{n} \frac{\tilde{X}_{t-1}}{\sqrt{k_{n}}} \frac{u_{t}}{\sqrt{k_{n}}} \frac{e^{\frac{1}{k_{n}} \sum_{j=1}^{t-1} c\left(\frac{j}{n}\right)}}{e^{\frac{1}{k_{n}} \sum_{j=1}^{n} c\left(\frac{j}{n}\right)}} \\
& =\sum_{t=1}^{n} \frac{\tilde{X}_{n}-\sum_{j=t}^{n} e^{-\frac{1}{k_{n}} \sum_{l=1}^{j} c\left(\frac{l}{n}\right)} u_{j}}{\sqrt{k_{n}}} \frac{u_{t}}{\sqrt{k_{n}}} e^{-\frac{1}{k_{n}} \sum_{j=t}^{n} c\left(\frac{j}{n}\right)} \\
& =\frac{\tilde{X}_{n}}{\sqrt{k_{n}}} \sum_{t=1}^{n} \frac{u_{t}}{\sqrt{k_{n}}} e^{-\frac{1}{k_{n}} \sum_{j=t}^{n} c\left(\frac{j}{n}\right)}-\sum_{t=1}^{n} \frac{u_{t}}{\sqrt{k_{n}}} e^{-\frac{1}{k_{n}} \sum_{j=t}^{n} c\left(\frac{j}{n}\right)} \sum_{j=t}^{n} e^{-\frac{1}{k_{n}} \sum_{l=1}^{j} c\left(\frac{l}{n}\right)} \frac{u_{j}}{\sqrt{k_{n}}} \\
& =: N_{n, 1}^{B}+N_{n, 2}^{B} .
\end{aligned}
$$

The first term of $(72)$ is

$N_{n, 1}^{B}=\frac{\tilde{X}_{n}}{\sqrt{k_{n}}} \sqrt{\frac{n}{k_{n}}} \int_{0}^{1} e^{-\frac{n}{k_{n}} \int_{s}^{1} c(a) d a} d B(s)\left[1+o_{p}(1)\right]=O_{p}\left(\sqrt{\frac{n}{k_{n}}}\right) \times \mathcal{N}\left(0, \frac{k_{n} \sigma^{2}}{2 n c(1)}\right)=O_{p}(1)$,

since 


$$
\mathbb{V}\left(\sqrt{\frac{n}{k_{n}}} \int_{0}^{1} e^{-\frac{n}{k_{n}} \int_{s}^{1} c(a) d a} d B(s)\right)=\sigma^{2} \frac{n}{k_{n}} \int_{0}^{1} e^{-\frac{2 n}{k_{n}} \int_{s}^{1} c(a) d a} d s \rightarrow \frac{\sigma^{2}}{2 c(1)},
$$

by Lemma 2 . The second term of $(72)$ is

$$
\begin{aligned}
& N_{n, 2}^{B}=-\frac{n}{k_{n}} \int_{0}^{1} e^{-\frac{n}{k_{n}} \int_{z}^{1} c(a) d a} \int_{z}^{1} e^{-\frac{n}{k_{n}} \int_{0}^{s} c(a) d a} d B(s) d B(z)\left[1+o_{p}(1)\right] \\
& =-e^{-\frac{n}{k_{n}} \int_{0}^{1} c(a) d a} \frac{n}{k_{n}} \int_{0}^{1} \int_{z}^{1} e^{-\frac{n}{k_{n}} \int_{z}^{s} c(a) d a} d B(s) d B(z)=O_{p}\left(\sqrt{\frac{n}{k_{n}}} e^{-\frac{n}{k_{n}} \int_{0}^{1} c(a) d a}\right),
\end{aligned}
$$

which is of smaller order than the term $N_{n, 1}^{B}$. Further, upon multiplication by $\frac{k_{n}}{n} e^{-\frac{1}{k_{n}} \sum_{j=1}^{n} c\left(\frac{j}{n}\right)}$ to match the scaling of the denominator and the first part of the numerator, both terms of $(72)$ are evidently of smaller order than (66) and $(70)$. It is convenient to employ this standardization in what follows and let

$$
\bar{N}_{n, 1}^{B}=\frac{k_{n}}{n} e^{-\frac{1}{k_{n}} \sum_{j=1}^{n} c\left(\frac{j}{n}\right)} N_{n, 1}^{B} \quad \text { and } \quad \bar{N}_{n, 2}^{B}=\frac{k_{n}}{n} e^{-\frac{1}{k_{n}} \sum_{j=1}^{n} c\left(\frac{j}{n}\right)} N_{n, 2}^{B} .
$$

(iii) Bias Calculation

Combining (66), 70), and (72), we obtain the following bias expression for centering the limit behavior of $\hat{\theta}_{O L S}$

$$
\begin{aligned}
k_{n}\left(\hat{\theta}_{O L S}-1\right) & =\frac{N_{n, 1}^{A}+O_{p}\left(\frac{k_{n}}{n} e^{-\frac{n}{k_{n}} \int_{0}^{1} c(a) d a}\right)}{D_{n, 1}+O_{p}\left(\frac{k_{n}}{n} e^{-\frac{n}{k_{n}} \int_{0}^{1} c(a) d a}\right)} \\
& =\frac{\frac{1}{n}\left(\frac{\tilde{X}_{n}}{\sqrt{k_{n}}}\right)^{2} \sum_{t=1}^{n} c\left(\frac{t}{n}\right) e^{-\frac{2}{k_{n}} \sum_{j=t}^{n} c\left(\frac{j}{n}\right)}+O_{p}\left(\frac{k_{n}}{n} e^{-\frac{n}{k_{n}} \int_{0}^{1} c(a) d a}\right)}{\frac{1}{n}\left(\frac{\tilde{X}_{n}}{\sqrt{k_{n}}}\right)^{2} \sum_{t=1}^{n} e^{-\frac{2}{k_{n}} \sum_{j=t}^{n} c\left(\frac{j}{n}\right)}+O_{p}\left(\frac{k_{n}}{n} e^{-\frac{n}{k_{n}} \int_{0}^{1} c(a) d a}\right)} \\
& =\frac{\frac{1}{n} \sum_{t=1}^{n} c\left(\frac{t}{n}\right) e^{-\frac{2}{k_{n}} \sum_{j=t}^{n} c\left(\frac{j}{n}\right)}}{\frac{1}{n} \sum_{t=1}^{n} e^{-\frac{2}{k_{n}} \sum_{j=t}^{n} c\left(\frac{j}{n}\right)}}\left(1+O_{p}\left(\frac{k_{n}}{n} e^{-\frac{n}{k_{n}} \int_{0}^{1} c(a) d a}\right)\right) \underset{n \rightarrow \infty}{\longrightarrow} c(1) .
\end{aligned}
$$

\section{(iv) Combining the Components of (65)}

Neglecting smaller order terms according to the earlier calculations of the respective orders of the elements of the numerator and denominator terms and using the notation $A \approx B$ when $A=B\left[1+o_{p}(1)\right]$ yields the asymptotic approximation 


$$
\begin{aligned}
& k_{n}\left(\hat{\theta}_{O L S}-1\right)-\frac{N_{n, 1}^{A}}{D_{n, 1}}=\frac{N_{n, 1}^{A}+N_{n, 2}^{A}+N_{n, 3}^{A}+\bar{N}_{n, 1}^{B}+\bar{N}_{n, 2}^{B}}{D_{n, 1}+D_{n, 2}+D_{n, 3}}-\frac{N_{n, 1}^{A}}{D_{n, 1}} \\
& =\frac{D_{n, 1}\left(N_{n, 2}^{A}+N_{n, 3}^{A}+\bar{N}_{n, 1}^{B}+\bar{N}_{n, 2}^{B}\right)-N_{n, 1}^{A}\left(D_{n, 2}+D_{n, 3}\right)}{D_{n, 1}\left(D_{n, 1}+D_{n, 2}+D_{n, 3}\right)} \\
& \approx \frac{N_{n, 2}^{A}+\bar{N}_{n, 1}^{B}}{D_{n, 1}}-\frac{N_{n, 1}^{A} D_{n, 2}}{D_{n, 1}^{2}} .
\end{aligned}
$$

Using (67), 68), (71) and (74), this recentred and scaled estimation error has the following explicit representation

$$
\begin{aligned}
& k_{n}\left(\hat{\theta}_{O L S}-1-\frac{\sum_{t=1}^{n} c\left(\frac{t}{n}\right) e^{-\frac{2}{k_{n}} \sum_{j=t}^{n} c\left(\frac{j}{n}\right)}}{k_{n} \sum_{t=1}^{n} e^{-\frac{2}{k_{n}} \sum_{j=t}^{n} c\left(\frac{j}{n}\right)}}\right) \approx\left[\frac{\frac{k_{n}}{n} e^{-\frac{1}{k_{n}} \sum_{j=1}^{n} c\left(\frac{j}{n}\right)} \tilde{X}_{n}}{\sqrt{k_{n}}} \sum_{t=1}^{n} \frac{u_{t}}{\sqrt{k_{n}}} e^{-\frac{1}{k_{n}} \sum_{j=t}^{n} c\left(\frac{j}{n}\right)}\right. \\
& \left.-\frac{2}{n} \frac{\tilde{X}_{n}}{\sqrt{k_{n}}} \sum_{t=1}^{n} c\left(\frac{t}{n}\right) e^{-\frac{2}{k_{n}} \sum_{j=t}^{n} c\left(\frac{j}{n}\right)} \sum_{j=t}^{n} e^{-\frac{1}{k_{n}} \sum_{l=1}^{j} c\left(\frac{l}{n}\right)} \frac{u_{j}}{\sqrt{k_{n}}}\right] /\left[\frac{1}{n}\left(\frac{\tilde{X}_{n}}{\sqrt{k_{n}}}\right)^{2} \sum_{t=1}^{n} e^{-\frac{2}{k_{n}} \sum_{j=t}^{n} c\left(\frac{j}{n}\right)}\right] \\
& +\left[\frac{1}{n}\left(\frac{\tilde{X}_{n}}{\sqrt{k_{n}}}\right)^{2} \sum_{t=1}^{n} c\left(\frac{t}{n}\right) e^{-\frac{2}{k_{n}} \sum_{j=t}^{n} c\left(\frac{j}{n}\right)} \times \frac{2}{n} \frac{\tilde{X}_{n}}{\sqrt{k_{n}}} \sum_{t=1}^{n} e^{-\frac{2}{k_{n}} \sum_{j=t}^{n} c\left(\frac{j}{n}\right)} \sum_{j=t}^{n} e^{-\frac{1}{k_{n}} \sum_{l=1}^{j} c(l / n)} \frac{u_{j}}{\sqrt{k_{n}}}\right] / \\
& {\left[\frac{1}{n}\left(\frac{\tilde{X}_{n}}{\sqrt{k_{n}}}\right)^{2} \sum_{t=1}^{n} e^{-\frac{2}{k_{n}} \sum_{j=t}^{n} c\left(\frac{j}{n}\right)}\right]^{2}} \\
& \approx\left[\sqrt{\frac{k_{n}}{n}} e^{-\frac{n}{k_{n}} \int_{0}^{1} c(a) d a} \int_{0}^{1} e^{-\frac{n}{k_{n}} \int_{s}^{1} c(a) d a} d B(s)\right. \\
& \left.-2 \sqrt{\frac{n}{k_{n}}} e^{-\frac{n}{k_{n}} \int_{0}^{1} c(a) d a} \int_{0}^{1} e^{-\frac{n}{k_{n}} \int_{s}^{1} c(a) d a} \int_{0}^{s} c(z) e^{-\frac{2 n}{k_{n}} \int_{z}^{s} c(a) d a} d z d B(s)\right] / \\
& {\left[\frac{\tilde{X}_{n}}{\sqrt{k_{n}}} \int_{0}^{1} e^{-\frac{2 n}{k_{n}} \int_{s}^{1} c(a) d a} d s\right]} \\
& +\left[2 \int_{0}^{1} c(s) e^{-\frac{2 n}{k_{n}} \int_{s}^{1} c(a) d a} d s \sqrt{\frac{n}{k_{n}}} e^{-\frac{n}{k_{n}} \int_{0}^{1} c(a) d a} \int_{0}^{1} e^{-\frac{n}{k_{n}} \int_{s}^{1} c(a) d a} \int_{0}^{s} e^{-\frac{2 n}{k_{n}} \int_{z}^{s} c(a) d a} d z d B(s)\right] \\
& /\left[\frac{\tilde{X}_{n}}{\sqrt{k_{n}}}\left(\int_{0}^{1} e^{-\frac{2 n}{k_{n}} \int_{s}^{1} c(a) d a} d s\right)^{2}\right]
\end{aligned}
$$

From Theorem 4. $\frac{\tilde{X}_{n}}{\sqrt{k_{n}}} \underset{n \rightarrow \infty}{\stackrel{d}{\longrightarrow}} X_{c(0)}={ }_{d} \mathcal{N}\left(0, \frac{\sigma^{2}}{2 c(0)}\right)$, and only early innovations with $t \sim 0$ influence the limit behavior of $\tilde{X}_{n}$ and, hence, the denominator of 76 ). By contrast, the 
numerator in (76) downweights early innovations and its limit behavior is governed as $n \rightarrow \infty$ by the right side of the unit interval, i.e. for values of $t \sim n$. Thus, numerator and denominator of (76) are independent as $n \rightarrow \infty$, both being Gaussian in the limit. Thus, the limit distribution of the ratio on the right side of $(76)$ is Cauchy $(\mathcal{C})$ and it remains to calculate the correct scaling factor of this distribution, which is determined by the respective variances of the Gaussian components.

As in earlier calculations

$$
\begin{gathered}
\int_{0}^{1} e^{-\frac{2 n}{k_{n}} \int_{s}^{1} c(a) d a} d s=\frac{k_{n}}{2 n c(1)}[1+o(1)], \\
\int_{0}^{1} \frac{1}{(2 c(s))^{2}} e^{-\frac{2 n}{k_{n}} \int_{s}^{1} c(a) d a} d s=\frac{k_{n}}{n} \frac{1}{(2 c(1))^{3}}[1+o(1)],
\end{gathered}
$$

and

$$
\int_{0}^{1} c(s) e^{-\frac{2 n}{k_{n}} \int_{s}^{1} c(a) d a} d s=\frac{k_{n}}{2 n}\left(1-e^{-\frac{2 n}{k_{n}} \int_{0}^{1} c(a) d a}\right)=\frac{k_{n}}{2 n}[1+o(1)] .
$$

Then, $X_{c(0)}\left(\int_{0}^{1} e^{-\frac{2 n}{k_{n}} \int_{s}^{1} c(a) d a} d s\right)^{2} \sim_{d} \frac{\sigma}{\sqrt{2 c(0)}} \mathcal{N}(0,1)\left(\frac{k_{n}}{2 n c(1)}\right)^{2}$ and by direct integration

$$
\begin{aligned}
& 2 \sqrt{\frac{n}{k_{n}}} \int_{0}^{1} e^{-\frac{n}{k_{n}} \int_{s}^{1} c(a) d a} \int_{0}^{s} c(z) e^{-\frac{2 n}{k_{n}} \int_{z}^{s} c(a) d a} d z d B(s) \\
& =\sqrt{\frac{k_{n}}{n}} \int_{0}^{1} e^{-\frac{n}{k_{n}} \int_{s}^{1} c(a) d a}\left(1-e^{-\frac{2 n}{k_{n}} \int_{0}^{s} c(a) d a}\right) d B(s) \approx \sqrt{\frac{k_{n}}{n}} \int_{0}^{1} e^{-\frac{n}{k_{n}} \int_{s}^{1} c(a) d a} d B(s) .
\end{aligned}
$$

We may therefore simplify the representation 76 as follows

$$
\begin{aligned}
& k_{n}\left(\hat{\theta}_{O L S}-1-\frac{\sum_{t=1}^{n} c\left(\frac{t}{n}\right) e^{-\frac{2}{k_{n}} \sum_{j=t}^{n} c\left(\frac{j}{n}\right)}}{k_{n} \sum_{t=1}^{n} e^{-\frac{2}{k_{n}} \sum_{j=t}^{n} c\left(\frac{j}{n}\right)}}\right) \\
& \approx\left[2 \int_{0}^{1} c(s) e^{-\frac{2 n}{k_{n}} \int_{s}^{1} c(a) d a} d s \sqrt{\frac{n}{k_{n}}} e^{-\frac{n}{k_{n}} \int_{0}^{1} c(a) d a} \int_{0}^{1} e^{-\frac{n}{k_{n}} \int_{s}^{1} c(a) d a} \int_{0}^{s} e^{-\frac{2 n}{k_{n}} \int_{z}^{s} c(a) d a} d z d B(s)\right] \\
& /\left[\frac{\tilde{X}_{n}}{\sqrt{k_{n}}}\left(\int_{0}^{1} e^{-\frac{2 n}{k_{n}} \int_{s}^{1} c(a) d a} d s\right)^{2}\right] \\
& \approx\left[\sqrt{\frac{k_{n}}{n}} e^{-\frac{n}{k_{n}} \int_{0}^{1} c(a) d a} \int_{0}^{1} e^{-\frac{n}{k_{n}} \int_{s}^{1} c(a) d a} \int_{0}^{s} e^{-\frac{2 n}{k_{n}} \int_{z}^{s} c(a) d a} d z d B(s)\right] /\left[\frac{\sigma}{\sqrt{2 c(0)}} \mathcal{N}(0,1)\left(\frac{k_{n}}{2 n c(1)}\right)^{2}\right]
\end{aligned}
$$




$$
\begin{aligned}
& \approx\left[\sqrt{\frac{k_{n}}{n}} e^{-\frac{n}{k_{n}} \int_{0}^{1} c(a) d a} \frac{k_{n}}{n} \int_{0}^{1} \frac{1}{2 c(s)} e^{-\frac{n}{k_{n}} \int_{s}^{1} c(a) d a} d B(s)\right] /\left[\frac{\sigma}{\sqrt{2 c(0)}} \mathcal{N}(0,1)\left(\frac{k_{n}}{2 n c(1)}\right)^{2}\right] \\
& =\left[e^{-\frac{n}{k_{n}} \int_{0}^{1} c(a) d a} \sqrt{\frac{n}{k_{n}}} \int_{0}^{1} \frac{1}{2 c(s)} e^{-\frac{n}{k_{n}} \int_{s}^{1} c(a) d a} d B(s)\right] /\left[\frac{\sigma}{\sqrt{2 c(0)}} \mathcal{N}(0,1)\left(\frac{1}{2 c(1)}\right)^{2}\right] \\
& \approx{ }_{d}\left[e^{-\frac{n}{k_{n}} \int_{0}^{1} c(a) d a} \frac{\sigma}{(2 c(1))^{\frac{3}{2}}} \mathcal{N}(0,1)\right] /\left[\frac{\sigma}{\sqrt{2 c(0)}}\left(\frac{1}{2 c(1)}\right)^{2} \mathcal{N}(0,1)\right] \\
& ={ }_{d} e^{-\frac{n}{k_{n}} \int_{0}^{1} c(a) d a} \times 2 \sqrt{c(0) c(1)} \times \mathcal{C} .
\end{aligned}
$$

As earlier, the representations involving $\int_{0}^{1} \frac{1}{2 c(s)} e^{-\frac{n}{k_{n}} \int_{s}^{1} c(a) d a} d B(s)$ in the above argument rely as $n \rightarrow \infty$ on the function $c(s)$ only in the immediate vicinity of $s=1$ and therefore $c(1)>0$ suffices for the asymptotics to hold.

We deduce the following asymptotic form of the centered estimator

$$
k_{n}\left(\hat{\theta}_{O L S}-1-\frac{\sum_{t=1}^{n} c\left(\frac{t}{n}\right) e^{-\frac{2}{k_{n}} \sum_{j=t}^{n} c\left(\frac{j}{n}\right)}}{k_{n} \sum_{t=1}^{n} e^{-\frac{2}{k_{n}} \sum_{j=t}^{n} c\left(\frac{j}{n}\right)}}\right) \approx 2 e^{-\frac{n}{k_{n}} \int_{0}^{1} c(a) d a} \sqrt{c(0) c(1)} \mathcal{C},
$$

which leads to the limit theory

$$
k_{n} e^{\frac{n}{k_{n}} \int_{0}^{1} c(a) d a}\left(\hat{\theta}_{O L S}-1-\frac{\sum_{t=1}^{n} c\left(\frac{t}{n}\right) e^{-\frac{2}{k_{n}} \sum_{j=t}^{n} c\left(\frac{j}{n}\right)}}{k_{n} \sum_{t=1}^{n} e^{-\frac{2}{k_{n}} \sum_{j=t}^{n} c\left(\frac{j}{n}\right)}}\right) \underset{n \rightarrow \infty}{\stackrel{d}{\longrightarrow}} 2 \sqrt{c(0) c(1)} \mathcal{C},
$$

as stated.

Finally, from 65 we have with random re-centering

$$
k_{n}\left(\hat{\theta}_{O L S}-1-\frac{\sum_{t=1}^{n} \frac{c(t / n)}{k_{n}} X_{t-1}^{2}}{\sum_{t=1}^{n} X_{t-1}^{2}}\right)=\frac{\frac{1}{n} \sum_{t=1}^{n} X_{t-1} u_{t}}{\frac{1}{n k_{n}} \sum_{t=1}^{n} X_{t-1}^{2}} .
$$

Then, using 66, 72, and the fact that

$$
\mathbb{V}\left(\sqrt{\frac{n}{k_{n}}} \int_{0}^{1} e^{-\frac{n}{k_{n}} \int_{s}^{1} c(a) d a} d B(s)\right)=\sigma^{2} \frac{n}{k_{n}} \int_{0}^{1} e^{-\frac{2 n}{k_{n}} \int_{s}^{1} c(a) d a} d s \rightarrow \frac{\sigma^{2}}{2 c(1)}
$$

we have

$$
\begin{aligned}
& k_{n} e^{\frac{n}{k_{n}} \int_{0}^{1} c(a) d a}\left(\hat{\theta}_{O L S}-1-\frac{\sum_{t=1}^{n} \frac{c(t / n)}{k_{n}} X_{t-1}^{2}}{\sum_{t=1}^{n} X_{t-1}^{2}}\right) \approx_{d} \frac{\frac{k_{n}}{n} N_{n, 1}^{B}}{D_{n, 1}} \\
& \approx_{d} \frac{\frac{k_{n}}{n} \sqrt{\frac{n}{k_{n}}} \int_{0}^{1} e^{-\frac{n}{k_{n}} \int_{s}^{1} c(a) d a} d B(s)}{\frac{\sigma}{\sqrt{2 c(0)}}\left(\frac{k_{n}}{2 n c(1)}\right) \mathcal{N}(0,1)} \\
& \underset{n \rightarrow \infty}{\stackrel{d}{\longrightarrow}} 2 \sqrt{c(0) c(1)} \mathcal{C} \text {, }
\end{aligned}
$$


as required.

\section{Proof of Theorem 6.}

Proof. We proceed in the same manner as for Theorem 4 and apply the martingale CLT. We first establish the stability condition for the variance of

$$
\frac{1}{\sqrt{k_{n}}} X_{\lfloor n r\rfloor}=\frac{1}{\sqrt{k_{n}}} \sum_{j=1}^{\lfloor n r\rfloor} e^{\frac{1}{k_{n}} \sum_{l=1}^{\lfloor n r\rfloor-j} c\left(\frac{\lfloor n r\rfloor-l+1}{n}\right)} u_{j}
$$

which has the form

$$
\begin{aligned}
\left\langle\frac{1}{\sqrt{k_{n}}} X_{\lfloor n r\rfloor}\right\rangle & =\frac{\sigma^{2}}{k_{n}} \sum_{j=1}^{\lfloor n r\rfloor} e^{\frac{2}{k_{n}} \sum_{l=1}^{\lfloor n r\rfloor-j} c\left(\frac{\lfloor n r\rfloor-l+1}{n}\right)} \\
& =\frac{\sigma^{2}}{k_{n}} \sum_{j=1}^{\lfloor n r\rfloor-\left\lfloor\sqrt{k_{n} n}\right\rfloor-1} \exp \left\{\frac{2}{k_{n}} \sum_{l=1}^{\lfloor n r\rfloor-j} c\left(\frac{\lfloor n r\rfloor-l+1}{n}\right)\right\} \\
& +\frac{\sigma^{2}}{k_{n}} \sum_{j=\lfloor n r\rfloor-\left\lfloor\sqrt{k_{n} n}\right\rfloor}^{\lfloor n r\rfloor} \exp \left\{\frac{2}{k_{n}} \sum_{l=1}^{\lfloor n r\rfloor-j} c\left(\frac{\lfloor n r\rfloor-l+1}{n}\right)\right\} .
\end{aligned}
$$

Note that when $j \leq\lfloor n r\rfloor-\left\lfloor\sqrt{k_{n} n}\right\rfloor$, we get $\lfloor n r\rfloor-j \geq\left\lfloor\sqrt{k_{n} n}\right\rfloor$, so that

$$
\exp \left\{\frac{2}{k_{n}} \sum_{l=1}^{\lfloor n r\rfloor-j} c\left(\frac{\lfloor n r\rfloor-l+1}{n}\right)\right\} \leq \exp \left\{-2 \sqrt{n / k_{n}} C\right\}
$$

and

$$
\begin{aligned}
& \frac{\sigma^{2}}{k_{n}} \sum_{j=1}^{\lfloor n r\rfloor-\left\lfloor\sqrt{k_{n}}\right\rfloor-1} \exp \left\{\frac{2}{k_{n}} \sum_{l=1}^{\lfloor n r\rfloor-j} c\left(\frac{\lfloor n r\rfloor-l+1}{n}\right)\right\} \\
& \leq \sigma^{2} \frac{\lfloor n r\rfloor-\sqrt{k_{n} n}}{k_{n}} \exp \left\{-2 \sqrt{n / k_{n}} C\right\} \underset{n \rightarrow \infty}{\longrightarrow} 0 .
\end{aligned}
$$

Now consider the second summation in $(78)$. If $j \geq\lfloor n r\rfloor-\sqrt{k_{n} n}$, we get $\frac{j+1}{n} \geq \frac{\lfloor n r\rfloor-\sqrt{k_{n} n}}{n} \approx$ $r$, so that $c\left(\frac{\lfloor n r\rfloor-l+1}{n}\right) \approx c(r)$ uniformly for $l=1, \ldots,\lfloor n r\rfloor-j$. Then, as in earlier 
calculations, we have

$$
\begin{aligned}
& \frac{\sigma^{2}}{k_{n}} \sum_{j=\lfloor n r\rfloor-\left\lfloor\sqrt{k_{n} n}\right\rfloor}^{\lfloor n r\rfloor} \exp \left\{\frac{2}{k_{n}} \sum_{l=1}^{\lfloor n r\rfloor-j} c\left(\frac{\lfloor n r\rfloor-l+1}{n}\right)\right\} \\
& \approx \frac{\sigma^{2}}{k_{n}} \sum_{j=\lfloor n r\rfloor-\left\lfloor\sqrt{k_{n} n}\right\rfloor}^{\lfloor n r\rfloor} \exp \left\{2 c(r) \frac{\lfloor n r\rfloor-j}{k_{n}}\right\} \\
& \approx \sigma^{2} \int_{0}^{\frac{\sqrt{k_{n} n}}{k_{n}}} e^{2 c(r) z} d z \underset{n \rightarrow \infty}{\longrightarrow} \sigma^{2} \int_{0}^{\infty} e^{2 c(r) z} d z=\frac{\sigma^{2}}{-2 c(r)} .
\end{aligned}
$$

Using (79) and (80) in 78 gives

$$
\left\langle\frac{1}{\sqrt{k_{n}}} X_{\lfloor n r\rfloor}\right\rangle \underset{n \rightarrow \infty}{\longrightarrow} \frac{\sigma^{2}}{-2 c(r)} .
$$

Next observe that for $n$ large enough and $s>r,\lfloor n r\rfloor<\lfloor n s\rfloor-\sqrt{k_{n} n}$, as $k_{n}$ goes to infinity slower than $n$. Hence, for $s>r$ the covariance

$$
\begin{aligned}
& \left\langle\frac{1}{\sqrt{k_{n}}} X_{\lfloor n r\rfloor}, \frac{1}{\sqrt{k_{n}}} X_{\lfloor n s\rfloor}\right\rangle \\
& =\frac{\sigma^{2}}{k_{n}} \sum_{j=1}^{\lfloor n r\rfloor} \exp \left\{\frac{1}{k_{n}}\left(\sum_{l=1}^{\lfloor n r\rfloor-j} c\left(\frac{\lfloor n r\rfloor-l+1}{n}\right)+\sum_{l=1}^{\lfloor n s\rfloor-j} c\left(\frac{\lfloor n s\rfloor-l+1}{n}\right)\right)\right\} \\
& \leq \frac{\sigma^{2}}{k_{n}} \sum_{j=1}^{\lfloor n s\rfloor-\sqrt{k_{n} n}} \exp \left\{\frac{1}{k_{n}}\left(\sum_{l=1}^{\lfloor n r\rfloor-j} c\left(\frac{\lfloor n r\rfloor-l+1}{n}\right)+\sum_{l=1}^{\lfloor n s\rfloor-j} c\left(\frac{\lfloor n s\rfloor-l+1}{n}\right)\right)\right\} \\
& \leq \frac{\sigma^{2}}{k_{n}} e^{-\sqrt{n / k_{n}} C} \sum_{j=1}^{\lfloor n s\rfloor-\sqrt{k_{n} n}} \exp \left\{\frac{1}{k_{n}} \sum_{l=1}^{\lfloor n r\rfloor-j} c\left(\frac{\lfloor n r\rfloor-l+1}{n}\right)\right\} \\
& \leq \frac{\sigma^{2}\left(\lfloor n s\rfloor-\sqrt{k_{n} n}\right)}{k_{n}} e^{-\sqrt{n / k_{n}} C} \underset{n \rightarrow \infty}{\longrightarrow} 0 .
\end{aligned}
$$

In precisely the same way as earlier in the proof of theorem 4 , it can be shown that the Lindeberg condition holds, thereby giving the finite dimensional convergence 


$$
\begin{aligned}
\frac{1}{\sqrt{k_{n}} X_{\lfloor n r\rfloor}}= & \frac{1}{\sqrt{k_{n}}} \sum_{j=1}^{\lfloor n r\rfloor} \exp \left\{\frac{1}{k_{n}} \sum_{l=1}^{\lfloor n r\rfloor-j} c\left(\frac{\lfloor n r\rfloor-l+1}{n}\right)\right\} u_{j} \\
& +\frac{1}{\sqrt{k_{n}}} \exp \left\{\frac{1}{k_{n}} \sum_{j=1}^{t} c(j / n)\right\} X_{0} \\
& \underset{n \rightarrow \infty}{d} \mathcal{N}\left(0, \frac{\sigma^{2}}{-2 c(r)}\right),
\end{aligned}
$$

and the stated result follows.

\section{REFERENCES}

Bykhovskaya, A. and P. C. B. Phillips, "Point Optimal Testing with Roots that are Functionally Local to Unity," submitted, 2017.

Chan, N. H. and C. Z. Wei, "Asymptotic inference for nearly nonstationary AR (1) processes," The Annals of Statistics, 1987, 15, 1050-1063.

Evans, G. W, "Pitfalls in testing for explosive bubbles in asset prices," The American Economic Review, 1991, 81 (4), 922-930.

Gao, J., I. Gijbels, and S. Van Bellegem, "Nonparametric simultaneous testing for structural breaks," Journal of Econometrics, 2008, 143 (1), 123-142.

Giraitis, L. and P. C. B. Phillips, "Uniform limit theory for stationary autoregression," Journal of time series analysis, 2006, 27 (1), 51-60.

, G. Kapetanios, and T. Yates, "Inference on stochastic time-varying coefficient models," Journal of Econometrics, 2014, 179 (1), 46-65.

Greenaway-McGrevy, R. and P. C. B. Phillips, "Hot property in New Zealand: Empirical evidence of housing bubbles in the metropolitan centres," New Zealand Economic Papers, 2016, 50 (1), 88-113.

Harvey, A. C., Forecasting, structural time series models and the Kalman filter, Cambridge university press, 1990.

Kristensen, D., "Non-parametric detection and estimation of structural change," The Econometrics Journal, 2012, 15 (3), 420-461.

Mikusheva, A., "Uniform inference in autoregressive models," Econometrica, 2007, 75 (5), 1411-1452.

, "One-Dimensional Inference in Autoregressive Models With the Potential Presence of a Unit Root," Econometrica, 2012, 80 (1), 173-212.

Phillips, P. C. B., "Towards a unified asymptotic theory for autoregression," Biometrika, 1987, 74 (3), 535-547. 
, "On Confidence Intervals for Autoregressive Roots and Predictive Regression," Econometrica, 2014, 82 (3), 1177-1195.

, "Uniform Inference in Nonparametric Time Varying Parameter Autoregression," Working Paper, Yale University, 2017.

and H. R Moon, "Linear regression limit theory for nonstationary panel data," Econometrica, 1999, 67 (5), 1057-1111.

and J. Yu, "Dating the timeline of financial bubbles during the subprime crisis," Quantitative Economics, 2011, 2 (3), 455-491.

and T. Magdalinos, "Limit theory for moderate deviations from a unit root," Journal of Econometrics, 2007, 136 (1), 115-130.

and _ _ "Unit root and cointegrating limit theory when initialization is in the infinite past," Econometric Theory, 2009, 25 (6), 1682-1715.

and V. Solo, "Asymptotics for Linear Processes," Annals of Statistics, 1992, 20, 971 .

and W. Ploberger, "Posterior odds testing for a unit root with data-based model selection," Econometric Theory, 1994, 10 (3-4), 774-808.

, S. Shuping, and J. Yu, "Testing for multiple bubbles: Historical episodes of exuberance and collapse in the S\&P 500," International Economic Review, 2015, 56 (4), 1043-1078.

_, , and __ _ "Testing For Multiple Bubbles: Limit Theory of RealTime Detectors," International Economic Review, 2015, 56 (4), 1079-1134.

, T. Magdalinos, and L. Giraitis, "Smoothing local-to-moderate unit root theory," Journal of Econometrics, 2010, 158 (2), 274-279.

, Y. Wu, and J. Yu, "Explosive behavior in the 1990s Nasdaq: When did exuberance escalate asset values?," International economic review, 2011, 52 (1), 201226.

Swamy, P. A. V. B., Statistical inference in random coefficient regression models, Vol. 55, Springer Science \& Business Media, 2012. 